QUARTERLY OF APPLIED MATHEMATICS

VOLUME LXVII, NUMBER 3

SEPTEMBER 2009, PAGES 441-475

S 0033-569X(09)01155-4

Article electronically published on May 6, 2009

\title{
ON THE STABILITY OF SHOCK WAVES IN LAYERED STRUCTURES AT THE PRESENCE OF THE ELECTRIC CURRENT
}

\author{
BY \\ A. M. BLOKHIN (Institute of Mathematics, Novosibirsk State University, Novosibirsk, 630090, \\ Russia) \\ AND \\ R. E. SEMENKO (Novosibisk State University, Novosibirsk, 630090, Russia)
}

\begin{abstract}
We are concerned with a hydrodynamical model of layered structures at the presence of the electric current. We formulate a linearized stability problem for shock waves and prove its ill-posedness, which means instability of shock waves for the given model of layered structures.
\end{abstract}

1. Introduction. It is known that the increase of oil reservoir performance is of great importance for modern power engineering. The difficulty in the resolution of this problem is that in chinked zones of exploiting collectors, there appear water-oil layered structures which block transport structures of collectors and, therefore, eject significant petroliferous areas from modes of water replacement. The restoration of the collector's permeability is only possible under the destruction of layered water-oil structures. As one of the possible mechanisms of destruction of such formations one can consider the parametric resonance arising under the harmonic long-time perturbation of the external boundary of a layered structure.

In [1], a system of hydrodynamical equations (in reversible and irreversible approximations) was proposed for gassy water-oil layered structures. In [2], some numerical results of the investigation of parametric instability were obtained in the framework of the model from [1].

However, already the first experimental studies of an opportunity for the organization of the parametric resonance by means of acoustic effect specify a problem of carrying the power action into the petroliferous collectors. In this connection, it should be noted that probably it is easier to provide the power action on a layered structure by using

Received February 12, 2008.

2000 Mathematics Subject Classification. Primary 35Q30, 83C22.

Key words and phrases. Layered structures, anisotropic dielectrics, electrohydrodynamical approximaton, linear problem, shock waves.

E-mail address: blokhin@math.nsc.ru

E-mail address: rsem86@mail.ru

(C) 2009 Brown University Reverts to public domain 28 years from publication 
methods of electrometry. The point is that water-oil layered structures are anisotropic dielectrics weakly conducting the electric current. Therefore, one can state the problem on the development of parametric instability in a layered structure at the presence of the small-amplitude alternating current.

The main goal of the present work is obtaining a system of hydrodynamical equations for gassy water-oil layered structures at the presence of the electric current with side (volume) charges. Moreover, we discuss the deduction of a simplified mathematical model of layered structures in the electrohydrodynamical approximation (see [3] for more details). At last, the main attention in the work is attracted to the stability analysis for shock waves for the electrohydrodynamical model of layered structures under consideration. Note that the possibility of organizing the parametric instability in water-oil layered systems at the presence of the electric current was discussed in [4, 5].

2. Preliminaries. Let us first briefly describe the system of hydrodynamical equations (in reversible approximation) for gassy water-oil layered structures and comment on the way of obtaining it. The basis of this method is continuum approximation. This approximation is described in detail in monograph [6] and is based on the validity of the conservation laws of mass, impulse, energy, and entropy. Considering a mixture of water, oil, and gas as a one-velocity layered continuum, a mathematical model of such layered structures was proposed in [1. This model consists of the following equations, which we write down in divergent form (in the form of conservation laws):

$$
\begin{gathered}
\rho_{t}+\operatorname{div}(\rho \boldsymbol{v})=0, \\
(\rho \mathbb{C})_{t}+\operatorname{div}(\rho \mathbb{C} \boldsymbol{v})=0, \\
\left(\rho v_{i}\right)_{t}+p_{x_{i}}+\sum_{k=1}^{3}\left(\rho v_{i} v_{k}-\bar{\sigma}_{i k}\right)_{x_{k}}=0, \quad i=1,2,3, \\
(\rho s)_{t}+\operatorname{div}(\rho s \boldsymbol{v})=0, \\
\left\{\rho\left(\varepsilon_{0}+\frac{|\boldsymbol{v}|^{2}}{2}\right)\right\}_{t}+\operatorname{div} \boldsymbol{Q}=0 .
\end{gathered}
$$

Here

$\rho$ is the density of the layered continuum,

$\boldsymbol{v}=\left(v_{1}, v_{2}, v_{3}\right)$ is the medium velocity vector,

$\mathbb{C}$ is the mass concentration of the gas,

$p$ is the pressure,

$s$ is the entropy,

$\varepsilon_{0}$ is the mass internal energy, and

$|\boldsymbol{v}|^{2}=(\boldsymbol{v}, \boldsymbol{v})$

The components $\bar{\sigma}_{i k}$ of the stress tensor and the vector $\boldsymbol{Q}=\left(Q_{1}, Q_{2}, Q_{3}\right)$ of the energy flux are described below.

To system (2.1a) - 2.1e) we add one more equation, which is obtained as follows. As the basis of the hydrodynamical model of the layered structures considered, we can put the well-known scheme of smectic hydrodynamical description (see [7, 8]). We 
describe alternating layers located one after another along the $x_{3}$-axis by the explicit assignment of an allocated element of the layered structure. Such an element is a surface in the space with coordinates $x_{1}, x_{2}, x_{3}$ :

$$
w(\boldsymbol{x}, t)=\theta, \quad \boldsymbol{x}=\left(x_{1}, x_{2}, x_{3}\right),
$$

where $\theta$ is the parameter identifying layers. The equation of the surface can be rewritten as

$$
\boldsymbol{x}=\boldsymbol{x}(\boldsymbol{u}, t, \theta) .
$$

Here $\boldsymbol{u}=\left(u_{1}, u_{2}\right)$ are the coordinates on the surface $\theta$. Therefore,

$$
w(\boldsymbol{x}(\boldsymbol{u}, t, \theta), t) \equiv \theta
$$

and

$$
\frac{d}{d t} w=\left\{\frac{\partial}{\partial t}+(\boldsymbol{v}, \nabla)\right\} w=0
$$

is the desired equation, where

$$
\boldsymbol{v}=\left.\frac{\partial \boldsymbol{x}}{\partial t}\right|_{\boldsymbol{u}, \theta}
$$

is the medium velocity vector (see above). In view of equation (2.1a), expression (2.2) can be rewritten in the divergent form

$$
(\rho w)_{t}+\operatorname{div}(\rho w \boldsymbol{v})=0 .
$$

Another fundamental fact underlying continuum approximation is formulated as follows. The thermodynamical state of a nonequilibrium system is defined by external and internal parameters of this system and the energy (see [6]). Also it is supposed that locally there exists a state function (nonequilibrium entropy). Therefore, to equations (2.1a)-(2.1e), (2.3) we should add the first law of thermodynamics, which for a layered structure has a complex form (see [1]):

$$
d \varepsilon_{0}=T d s-p d V+V\{(\boldsymbol{f}, d \boldsymbol{b})+Z d \mathbb{C}+\Phi d(\triangle w)+(\boldsymbol{\xi}, d \boldsymbol{a})\},
$$

where $T$ is the temperature,

$V\left(=\frac{1}{\rho}\right)$ is the specific volume,

$\boldsymbol{b}=\left(b_{1}, b_{2}, b_{3}\right)=\nabla w$

$\varepsilon_{0}=e_{0}+V E_{0}$,

$e_{0}=e_{0}(V, s, \mathbb{C})$ is the state equation,

$$
\begin{gathered}
E_{0}=\left\{\frac{B}{8}\left(\frac{|\boldsymbol{b}|^{2}}{q^{2}}-1\right)^{2}+\frac{N}{2} \frac{(\triangle w)^{2}}{q^{2}}\right. \\
\left.+\frac{\alpha\left(\sigma^{\prime}\right)^{2}}{2 q^{2}}|\boldsymbol{b}|^{2}\left((\boldsymbol{d}, \boldsymbol{a})^{2}-|\boldsymbol{a}|^{2}\right)+\sigma q\left(\frac{1}{d_{3}}-1\right)\right\}
\end{gathered}
$$

is the volume density of the "surface elastic energy" of a deformable layered structure (see $([1,7,8])$,

$B, N(>0), \alpha(<0)$ are some constants,

$q=q(V, s)$ is the density of the structure's layer,

$\boldsymbol{d}=\left(d_{1}, d_{2}, d_{3}\right)=\frac{\boldsymbol{b}}{\mid \boldsymbol{b}}$,

$\boldsymbol{a}=\left(a_{1}, a_{2}, a_{3}\right)=\nabla \mathbb{C}$, 
$\sigma=\sigma(\mathbb{C})(\geqslant 0)$ is the "surface tension",

$$
\begin{gathered}
\boldsymbol{f}=\left(f_{1}, f_{2}, f_{3}\right)=\frac{B}{2 q^{2}}\left(\frac{|\boldsymbol{b}|^{2}}{q^{2}}-1\right) \boldsymbol{b}+\frac{\alpha\left(\sigma^{\prime}\right)^{2}}{q^{2}}\left((\boldsymbol{b}, \boldsymbol{a})-|\boldsymbol{a}|^{2} \boldsymbol{b}\right)+\frac{\sigma q}{b_{3}}\left(d_{1}, d_{2}, \frac{-d_{1}^{2}-d_{2}^{2}}{d_{3}}\right), \\
Z=\rho\left(e_{0}\right)_{\mathbb{C}}+\frac{\alpha \sigma^{\prime} \sigma^{\prime \prime}}{q^{2}}|\boldsymbol{b}|^{2}\left((\boldsymbol{d}, \boldsymbol{a})^{2}-|\boldsymbol{a}|^{2}\right)+\sigma^{\prime} q\left(\frac{1}{d_{3}}-1\right), \\
\Phi=\frac{N}{q^{2}} \triangle w,
\end{gathered}
$$

$\triangle$ is the Laplace operator, $\triangle=\sum_{k=1}^{3} \frac{\partial^{2}}{\partial x_{k}^{2}}$,

$$
\begin{gathered}
\boldsymbol{\xi}=\left(\xi_{1}, \xi_{2}, \xi_{3}\right)=\frac{\alpha\left(\sigma^{\prime}\right)^{2}}{q^{2}}|\boldsymbol{b}|^{2}((\boldsymbol{d}, \boldsymbol{a}) \boldsymbol{d}-\boldsymbol{a}), \\
T=\left(e_{0}\right)_{s}+V\left(E_{0}\right)_{s}, \\
p=-\left(e_{0}\right)_{V}-E_{0}-V\left(E_{0}\right)_{V}, \\
c_{s}^{2}=p_{\rho}=-V^{2} p_{V}=-V^{2}\left\{\left(e_{0}\right)_{V V}+2\left(E_{0}\right)_{V}+V\left(E_{0}\right)_{V V}\right\}
\end{gathered}
$$

is the square of the sound velocity (see [9]),

$$
\begin{gathered}
\left(E_{0}\right)_{\beta}=-\frac{q_{\beta}}{q^{3}}\left\{\frac{B}{2}\left(\frac{|\boldsymbol{b}|^{2}}{q^{2}}-1\right)|\boldsymbol{b}|^{2}+N(\triangle w)^{2}\right. \\
\left.+\alpha\left(\sigma^{\prime}\right)^{2}|\boldsymbol{b}|^{2}\left((\boldsymbol{d}, \boldsymbol{a})^{2}-|\boldsymbol{a}|^{2}\right)\right\}+\sigma q_{\beta}\left(\frac{1}{d_{3}}-1\right), \quad \beta=V, s, \\
\left(E_{0}\right)_{V V}=\left(3 \frac{q_{V}^{2}}{q^{4}}-\frac{q_{V V}}{q^{3}}\right)\left\{\frac{B}{2}\left(\frac{|\boldsymbol{b}|^{2}}{q^{2}}-1\right)|\boldsymbol{b}|^{2}+N(\triangle w)^{2}+\alpha\left(\sigma^{\prime}\right)^{2}|\boldsymbol{b}|^{2}\left((\boldsymbol{d}, \boldsymbol{a})^{2}-|\boldsymbol{a}|^{2}\right)\right\} \\
+B|\boldsymbol{b}|^{4} \frac{q_{V}^{2}}{q^{6}}+\sigma q_{V V}\left(\frac{1}{d_{3}}-1\right) .
\end{gathered}
$$

REMARK 2.1. The first two terms in formula (2.5) describe the deformation of layered structure and are intrinsical for smectic description (see [7, 8]). The next two terms are due to the dependence of the "surface tension" at the interface of the layers on the concentration $\mathbb{C}$ of the gas in the system. From the physical point of view it is clear that the third term provides additional tangential stresses caused by the presence of gradients of the "surface tension", and the fourth one corresponds to a change in the energy of the system caused by a change in the area of the layer in the process of deformation.

Note that equations (2.1a) (2.1d) in a nonconservative form appear as follows:

$$
\begin{gathered}
\frac{d \rho}{d t}+\rho \operatorname{div} \boldsymbol{v}=0, \\
\frac{d \mathbb{C}}{d t}=0, \\
\frac{d v_{i}}{d t}+V p_{x_{i}}=V \sum_{k=1}^{3}\left(\bar{\sigma}_{i k}\right)_{x_{k}}, \quad i=1,2,3, \\
\frac{d s}{d t}=0 .
\end{gathered}
$$


Using relations (2.6), (2.7), one can take the following equation instead of (2.8a):

$$
\frac{d p}{d t}+\rho c_{s}^{2} \operatorname{div} \boldsymbol{v}=-\left(\boldsymbol{f}+V\left(E_{0}\right)_{V \boldsymbol{b}}, \frac{d \boldsymbol{b}}{d t}\right)-\left(1-2 \frac{q_{V}}{q} V\right)\left\{\left(\boldsymbol{\xi}, \frac{d \boldsymbol{a}}{d t}\right)+\Phi \frac{d(\triangle w)}{d t}\right\} .
$$

Here

$$
\begin{gathered}
\left(E_{0}\right)_{V \boldsymbol{b}}=\left(\left(E_{0}\right)_{V b_{1}},\left(E_{0}\right)_{V b_{2}},\left(E_{0}\right)_{V b_{3}}\right)=-\frac{q_{V}}{q^{3}}\left\{B\left(2 \frac{|\boldsymbol{b}|^{2}}{q^{2}}-1\right) \boldsymbol{b}\right. \\
\left.+2 \alpha\left(\sigma^{\prime}\right)^{2}\left((\boldsymbol{b}, \boldsymbol{a}) \boldsymbol{a}-|\boldsymbol{a}|^{2} \boldsymbol{b}\right)\right\}+\frac{\sigma q_{V}}{b_{3}}\left(d_{1}, d_{2}, \frac{-d_{1}^{2}-d_{2}^{2}}{d_{3}}\right) .
\end{gathered}
$$

Moreover, by means of equations (2.2), (2.8b), the terms

$$
\frac{d \boldsymbol{b}}{d t}, \quad \frac{d \boldsymbol{a}}{d t}, \quad \frac{d(\triangle w)}{d t},
$$

appearing on the right-hand side of equation (2.9) are defined as

$$
\begin{gathered}
\frac{d \boldsymbol{b}}{d t}=-(\boldsymbol{b}, \nabla) \boldsymbol{v}-[\boldsymbol{b}, \operatorname{curl} \boldsymbol{v}], \quad \operatorname{curl} \boldsymbol{b}=0, \\
\frac{d \boldsymbol{a}}{d t}=-(\boldsymbol{a}, \nabla) \boldsymbol{v}-[\boldsymbol{a}, \operatorname{curl} \boldsymbol{v}], \quad \operatorname{curl} \boldsymbol{a}=0, \\
\frac{d(\triangle w)}{d t}=-2 \sum_{k=1}^{3}\left(\frac{\partial \boldsymbol{v}}{d x_{k}}, \nabla\right) b_{k}-(\boldsymbol{b}, \triangle \boldsymbol{v}) .
\end{gathered}
$$

Until now we did not present an explicit form of the components $\bar{\sigma}_{i k}$ of the stress tensor and the vector of energy flux $\boldsymbol{Q}$. Note that, along with the statement that conservation laws are satisfied and the density of the internal energy depends on the thermodynamic degrees of freedom, the physical content of the reversible theory is determined by the form of a stress tensor $\bar{\sigma}_{i k}$. Let us show that in reversible theory this form is uniquely determined by the conservation laws (2.1a)-(2.1e), (2.3), and the first law of thermodynamics (2.4). Indeed, as usual in hydrodynamics, the law of energy conservation (2.1e) should be a consequence of all the other conservation laws. Following [6], we differentiate with respect to the time the full energy

$$
E=\rho \frac{|\boldsymbol{v}|^{2}}{2}+\rho \varepsilon_{0}
$$

and, by virtue of (2.4), obtain

$$
\begin{gathered}
\frac{\partial E}{d t}=\left(\varepsilon_{0}+p V+\frac{|\boldsymbol{v}|^{2}}{2}\right) \frac{\partial \rho}{\partial t}+\left(\rho \boldsymbol{v}, \frac{\partial \boldsymbol{v}}{\partial t}\right)+\rho T \frac{\partial s}{d t}+\left(\boldsymbol{f}, \frac{\partial \boldsymbol{b}}{\partial t}\right) \\
+Z \frac{\partial \mathbb{C}}{\partial t}+\Phi \frac{(\partial \triangle w)}{\partial t}+\left(\boldsymbol{\xi}, \frac{\partial \boldsymbol{a}}{\partial t}\right) .
\end{gathered}
$$

Substituting into the right-hand side of expression (2.11) the derivatives

$$
\frac{\partial \rho}{\partial t}, \quad \frac{\partial \boldsymbol{v}}{\partial t}, \quad \frac{\partial s}{\partial t}, \quad \frac{\partial \boldsymbol{b}}{\partial t}, \quad \frac{\partial \mathbb{C}}{\partial t}, \quad \frac{\partial(\triangle w)}{\partial t}, \quad \frac{\partial \boldsymbol{a}}{\partial t},
$$

which are found from (2.8a) $-(2.8 \mathrm{~d}),(2.10 \mathrm{a})-(2.10 \mathrm{c})$, after simple but bulky calculations, from (2.11) we obtain the law of energy conservation (2.1e). Doing so we define the 
vector of energy flux $\boldsymbol{Q}$ and the components $\bar{\sigma}_{i k}$ of the stress tensor as follows:

$$
\begin{gathered}
\bar{\sigma}_{i k}=b_{i}\left(-f_{k}+\frac{\partial \Phi}{\partial x_{k}}\right)-a_{i} \xi_{k}-\Phi B_{i k}, \quad i, k=1,2,3, \\
\boldsymbol{Q}=\rho \boldsymbol{v}\left\{\varepsilon_{0}+p V+\frac{|\boldsymbol{v}|^{2}}{2}\right\}+\boldsymbol{q}, \quad \boldsymbol{q}=\left(q_{1}, q_{2}, q_{3}\right), \\
q_{k}=\left(f_{k}-\frac{\partial \Phi}{\partial x_{k}}\right)(\boldsymbol{b}, \boldsymbol{v})+\xi_{k}(\boldsymbol{a}, \boldsymbol{v})+\Phi \sum_{l=1}^{3}\left(v_{l} B_{k l}+b_{l} \frac{\partial v_{l}}{\partial x_{k}}\right), k=1,2,3,
\end{gathered}
$$

where

$$
B_{i k}=\frac{\partial^{2} w}{\partial x_{i} \partial x_{k}} .
$$

Thus, the system of conservation laws (2.1a)-(2.1d), (2.3) describes the reversible hydrodynamic motion of a layered structure. The law of energy conservation (2.1e) is a consequence of all equations of this system. The proposed ideal hydrodynamics of a layered structure can be a basis for the introduction of thermodynamic irreversibility in the motion equations (see [1]).

REMARK 2.2. In [10, 11], conditions on a surface of strong discontinuity for layered structures were obtained, a linearized stability problem for shock waves was formulated and its ill-posedness was proved (this means the instability of shock waves for the hydrodynamical model described above).

3. Hydrodynamics of an anisotropic layered dielectric. Taking the equations of reversible hydrodynamics of layered structures described in the previous section as a basis, we show how to obtain a closed system connecting electrodynamics and mechanics of anisotropic layered dielectrics to within terms of order $\frac{1}{c^{2}}$, where $c$ is the speed of light.

First of all, we observe that processes of polarization and magnetization typical for anisotropic dielectrics lead the following modified version of the first law of thermodynamics (2.4) (see [12]):

$$
\begin{aligned}
& d \varepsilon_{0}=T d s-p d V+\left(\boldsymbol{E}^{\prime}, d \mathcal{P}\right)-\left(\boldsymbol{m}^{\prime}, d \boldsymbol{B}\right) \\
& +V\{(\boldsymbol{f}, d \boldsymbol{b})+Z d \mathbb{C}+\Phi d(\triangle w)+(\boldsymbol{\xi}, d \boldsymbol{a})\}
\end{aligned}
$$

Here

$\varepsilon_{0}=e_{0}+V E_{0}$ is the internal energy,

$E_{0}$ is defined by (2.5),

$e_{0}=e_{0}(V, s, \mathbb{C}, \mathcal{P}, \boldsymbol{B})$ is the state equation,

$\boldsymbol{E}=\left(E_{1}, E_{2}, E_{3}\right)$ is the electric force (in the laboratory reference frame),

$\boldsymbol{E}^{\prime}=\left(\boldsymbol{E}+\frac{1}{c}[\boldsymbol{v}, \boldsymbol{B}]\right)$ is the electric force in the rest frame of a liquid particle of the medium,

$\boldsymbol{B}=\left(B_{1}, B_{2}, B_{3}\right)$ is the vector of magnetic induction (in the laboratory reference frame), $\boldsymbol{B}^{\prime}=\left(\boldsymbol{B}-\frac{1}{c}[\boldsymbol{v}, \boldsymbol{E}]\right)$ is the vector of magnetic induction in the rest frame,

$\boldsymbol{P}$ is the vector of electric polarization (in the laboratory reference frame),

$\mathcal{P}=V \boldsymbol{P}$,

$\boldsymbol{M}$ is the magnetization of the medium (in the laboratory reference frame), 
$\boldsymbol{M}^{\prime}=\left(\boldsymbol{M}+\frac{1}{c}[\boldsymbol{v}, \boldsymbol{P}]\right)$ is the magnetization of the medium in the rest frame, and $\boldsymbol{m}=V \boldsymbol{M}, \boldsymbol{m}^{\prime}=V \boldsymbol{M}^{\prime}$.

REMARK 3.1. In (3.1) it is already taken into account that (see [12])

$$
\begin{gathered}
\boldsymbol{P}^{\prime}=\boldsymbol{P} \\
|\boldsymbol{M}| \sim \frac{1}{c},
\end{gathered}
$$

where $\boldsymbol{P}^{\prime}$ is the vector of electric polarization in the rest frame.

Let us now write down the system of Maxwell's equations at the presence of volume charges (see [12]):

$$
\begin{aligned}
\operatorname{curl} \boldsymbol{E}= & -\frac{1}{c} \frac{\partial \boldsymbol{B}}{\partial t}, \quad \operatorname{curl} \boldsymbol{H}=-\frac{1}{c} \frac{\partial \boldsymbol{D}}{\partial t}+\frac{4 \pi}{c} \boldsymbol{j}, \\
& \operatorname{div} \boldsymbol{D}=4 \pi \rho_{e}, \quad \operatorname{div} \boldsymbol{B}=0 .
\end{aligned}
$$

Here

$\boldsymbol{D}=\left(D_{1}, D_{2}, D_{3}\right)=(\boldsymbol{E}+4 \pi \boldsymbol{P})$ is the vector of electric induction (in the laboratory reference frame),

$\boldsymbol{H}=\left(H_{1}, H_{2}, H_{3}\right)=(\boldsymbol{B}-4 \pi \boldsymbol{M})$ is the vector of the magnetic field (in the laboratory reference frame),

$\boldsymbol{j}=\left(j_{1}, j_{2}, j_{3}\right)$ the vector of current density, and

$\rho_{e}$ is the volume charge density.

REMARK 3.2. The last two equations in system (3.2) can be considered as additional restrictions on the initial data. This becomes obvious if we add the law of volume charge conservation

$$
\frac{\partial \rho_{e}}{\partial t}+\operatorname{div} \boldsymbol{j}=0
$$

to (3.2).

Before passing to the construction of the system of conservation laws connecting the electrodynamics and mechanics of layered anisotropic dielectrics, we present two relations obtained from Maxwell's equations by simple but bulky calculations. The first of them is an expression for ponderomotive forces (i.e., forces acting from the side of an electromagnetic field to a layered structure under polarization and magnetization; see ([12]). This relation is given in the form

$$
\begin{gathered}
\frac{1}{4 \pi c}\left([\boldsymbol{E}, \boldsymbol{B}]_{i}\right)_{t}=\frac{1}{c}\left([\boldsymbol{B}, \boldsymbol{P}]_{i}\right)_{t}-\left(\boldsymbol{P}, \boldsymbol{E}_{x_{i}}\right)-\left(\boldsymbol{M}, \boldsymbol{B}_{x_{i}}\right)-\frac{1}{c}[\boldsymbol{j}, \boldsymbol{B}]_{i} \\
+\sum_{k=1}^{3}\left\{\frac{B_{k} H_{i}+D_{k} E_{i}}{4 \pi}-\left(\frac{|\boldsymbol{E}|^{2}+|\boldsymbol{B}|^{2}}{8 \pi}-(\boldsymbol{B}, \boldsymbol{M})\right) \delta_{i k}\right\}_{x_{k}}-\rho_{e} E_{i}, i=1,2,3,
\end{gathered}
$$

where $[\boldsymbol{E}, \boldsymbol{B}]_{i}$ is the $i$ th component of the vector product $[\boldsymbol{E}, \boldsymbol{B}]$, etc. The second relation is the Umow-Pointing equation (see [12]) (it describes an exchange of energy between the electromagnetic field and the medium)

$$
\frac{\partial}{\partial t}\left(\frac{|\boldsymbol{E}|^{2}+|\boldsymbol{B}|^{2}}{8 \pi}\right)=-\frac{c}{4 \pi} \operatorname{div}([\boldsymbol{E}, \boldsymbol{H}])-(\boldsymbol{j}, \boldsymbol{E})-\left(\boldsymbol{E}, \boldsymbol{P}_{t}\right)+\left(\boldsymbol{M}, \boldsymbol{B}_{t}\right) .
$$


We now begin to construct the above-mentioned system of conservation laws. Instead of equations (2.1a) $-2.1 \mathrm{~d}$ ) we take

$$
\begin{gathered}
\rho_{t}+\operatorname{div}(\rho \boldsymbol{v})=0 \\
(\rho \mathbb{C})_{t}+\operatorname{div}(\rho \mathbb{C} \boldsymbol{v})=0 \\
\left\{\rho v_{i}+\frac{1}{4 \pi c}[\boldsymbol{E}, \boldsymbol{B}]_{i}\right\}_{t}+\left\{p+\frac{|\boldsymbol{E}|^{2}+|\boldsymbol{B}|^{2}}{8 \pi}-(\boldsymbol{B}, \boldsymbol{M})\right\}_{x_{i}} \\
+\sum_{k=1}^{3}\left(\rho v_{i} v_{k}-\bar{\sigma}_{i k}-\frac{H_{i} B_{k}+E_{i} D_{k}}{4 \pi}+\Sigma_{i k}\right)_{x_{k}}=0, i=1,2,3, \\
(\rho s)_{t}+\operatorname{div}(\rho s \boldsymbol{v})=\frac{R}{T} .
\end{gathered}
$$

Here $R$ is the dissipative function (in connection with the presence of the current density vector $j$ in Maxwell's equations we should include the entropy production in the righthand side of the law of entropy conservation); an explicit form of $\Sigma_{i k}$ and the dissipative function $R$ will be described below.

Let us calculate the time derivative of the full energy

$$
\mathcal{E}=\rho \frac{|\boldsymbol{v}|^{2}}{2}+\rho \varepsilon_{0}+\frac{|\boldsymbol{E}|^{2}+|\boldsymbol{B}|^{2}}{8 \pi} .
$$

In view of (3.1),

$$
\begin{gathered}
\frac{\partial \mathcal{E}}{\partial t}=\frac{\partial}{\partial t}\left(\frac{|\boldsymbol{E}|^{2}+|\boldsymbol{B}|^{2}}{8 \pi}\right)+\left(\varepsilon_{0}+p V+\frac{|\boldsymbol{v}|^{2}}{2}-\left(\boldsymbol{E}^{\prime}, \mathcal{P}\right)\right) \frac{\partial \rho}{\partial t} \\
+\left(\rho \boldsymbol{v}, \frac{\partial \boldsymbol{v}}{\partial t}\right)+\rho T \frac{\partial s}{\partial t}+\left(\boldsymbol{f}, \frac{\partial \boldsymbol{b}}{\partial t}\right)+Z \frac{\partial \mathbb{C}}{\partial t}+\Phi \frac{\partial(\triangle w)}{\partial t} \\
+\left(\boldsymbol{\xi}, \frac{\partial a}{\partial t}\right)+\left(\boldsymbol{E}^{\prime}, \frac{\partial \boldsymbol{P}}{\partial t}\right)-\left(\boldsymbol{M}^{\prime}, \frac{\partial \boldsymbol{B}}{\partial t}\right) .
\end{gathered}
$$

Substituting the derivatives

$$
\frac{\partial \rho}{\partial t}, \quad \frac{\partial \boldsymbol{v}}{\partial t}, \quad \frac{\partial s}{\partial t}, \quad \frac{\partial \boldsymbol{b}}{\partial t}, \quad \frac{\partial \mathbb{C}}{\partial t}, \quad \frac{\partial(\triangle w)}{\partial t}, \quad \frac{\partial \boldsymbol{a}}{\partial t}
$$

found from (3.6a)-(3.6d) and (2.10a $)-(2.10 \mathrm{c})$ into (3.7) and using relations (3.4), (3.5), after cumbersome calculations from (3.7), we get the law of conservation of energy

$$
\frac{\partial \mathcal{E}}{\partial t}+\operatorname{div}\left(\boldsymbol{Q}-(\boldsymbol{E}, \boldsymbol{P}) \boldsymbol{v}+\frac{c[\boldsymbol{E}, \boldsymbol{H}]}{4 \pi}\right)=0
$$

if $\Sigma_{i k}$ and the dissipative function $R$ are defined as follows:

$$
\begin{gathered}
\Sigma_{i k}=[\boldsymbol{B}, \boldsymbol{P}]_{i} \frac{v_{k}}{c}, \quad i, k=1,2,3, \\
R=\left(\left(\boldsymbol{j}-\rho_{e} \boldsymbol{v}\right), \boldsymbol{E}^{\prime}\right) .
\end{gathered}
$$

The components $\bar{\sigma}_{i k}$ of the stress tensor and the energy flux $\boldsymbol{Q}$ were defined in Section 1.

The expression for the dissipative function $R$ (see (3.9)) introduces Ohm's law for moving dielectrics with outflow:

$$
\boldsymbol{j}=\rho_{e}\left(\boldsymbol{v}+\boldsymbol{E}^{\prime} \Omega\right),
$$


where $\Omega=\left(\Omega_{i k}\right), i, k=1,2,3$, is the conductivity tensor (or the mobility tensor, see [3]), and we shall suppose that its structure corresponds to smectic symmetry (see [8]):

$$
\Omega=\omega_{\perp} I_{3}+\left(\omega_{\|}-\omega_{\perp}\right) \mathcal{D}
$$

$I_{3}$ is the unitary matrix of the third order,

$\mathcal{D}=\left(d_{i} d_{k}\right), i, k=1,2,3$,

$\omega_{\|}, \omega_{\perp}$ are some constants.

By virtue of (3.9) and (3.10), we finally get the following expression for the dissipative function $R$ :

$$
R=\rho_{e}\left(\boldsymbol{E}^{\prime} \Omega, \boldsymbol{E}^{\prime}\right),
$$

where $\Omega>0$ if $R>0$.

Thus, the system of conservation laws (3.6a) -3.6d), (2.3), (3.2) represents the full system of electrohydrodynamical equations for liquid dielectrics, and the law of energy conservation (3.8) is a consequence of this system.

On smooth solutions the above formulated system of conservation laws can be reduced to a nonconservative form. After simple but long calculations we obtain this nonconservative form:

$$
\begin{gathered}
\frac{d \rho}{d t}+\rho \operatorname{div} \boldsymbol{v}=0 \quad\left(\text { or } \frac{d V}{d t}-V \operatorname{div} \boldsymbol{v}=0\right), \\
\frac{d w}{d t}=0, \\
\frac{d s}{d t}=\frac{R V}{T}, \\
\frac{d \mathbb{C}}{d t}=0, \\
\frac{d}{d t}\left\{v_{i}+\frac{[\boldsymbol{B}, \mathcal{P}]_{i}}{c}\right\}+V p_{x_{i}}=V \sum_{k=1}^{3}\left(\bar{\sigma}_{i k}\right)_{x_{k}}+\left(\mathcal{P}, \boldsymbol{E}_{x_{i}}\right) \\
+\left(\boldsymbol{m}, B_{x_{i}}\right)+\frac{1}{c} V[\boldsymbol{j}, \boldsymbol{B}]_{i}+\rho_{e} V E_{i}, \quad i=1,2,3 .
\end{gathered}
$$

We note that in the motion equations (3.13e) obtained from the vector law of impulse conservation there is a ponderomotive force (more precisely, its components) which at the presence of polarization and magnetization differs from the Lorentz force

$$
\rho_{e} \boldsymbol{E}+\frac{1}{c}[\boldsymbol{j}, \boldsymbol{B}] .
$$

As in Section 1, it follows from (3.1) that

$$
\begin{gathered}
p=-\left(e_{0}\right)_{V}-E_{0}-V\left(E_{0}\right)_{V}, \\
c_{s}^{2}=p_{\rho}=-V^{2} p_{V}=V^{2}\left\{\left(e_{0}\right)_{V V}+2\left(E_{0}\right)_{V}+V\left(E_{0}\right)_{V V}\right\} .
\end{gathered}
$$

Using these relations, we can replace (3.13a) with the equation for the pressure $p$ :

$$
\begin{gathered}
\frac{d p}{d t}+\rho c_{s}^{2} \operatorname{div} \boldsymbol{v}=p_{s} \frac{R V}{T}-\left(\boldsymbol{f}+V\left(E_{0}\right)_{V \boldsymbol{b}}, \frac{d \boldsymbol{b}}{d t}\right) \\
-\left(1-2 \frac{q_{V}}{q} V\right)\left\{\left(\boldsymbol{\xi}, \frac{d \boldsymbol{a}}{d t}\right)+\Phi \frac{d(\triangle w)}{d t}\right\}-\left(\left(e_{0}\right)_{V \mathcal{P}}, \frac{d \mathcal{P}}{d t}-\left(\left(e_{0}\right)_{V \boldsymbol{B}}, \frac{d \boldsymbol{B}}{d t}\right) .\right.
\end{gathered}
$$


Here

$$
\begin{gathered}
p_{s}=-\left(e_{0}\right)_{V s}-\left(E_{0}\right)_{s}-V\left(E_{0}\right)_{V s}, \\
\left(E_{0}\right)_{V s}=\left(3 \frac{q_{V} q_{s}}{q^{4}}-\frac{q_{V s}}{q^{3}}\right)\left\{\frac{B}{2}\left(\frac{|\boldsymbol{b}|^{2}}{q^{2}}-1\right)|\boldsymbol{b}|^{2}+N(\Delta w)^{2}\right. \\
\left.+\alpha\left(\sigma^{\prime}\right)^{2}|\boldsymbol{b}|^{2}\left((\boldsymbol{d}, \boldsymbol{a})^{2}-|\boldsymbol{a}|^{2}\right)\right\}+B|\boldsymbol{b}|^{4} \frac{q_{V} q_{s}}{q^{6}}+\sigma q_{V s}\left(\frac{1}{d_{3}}-1\right) .
\end{gathered}
$$

4. Mathematical model of layered dielectrics in approximation. The mathematical model described in the previous section is rather complicated and below we shall consider its simplified version corresponding to electrohydrodynamical approximation (see [3]) by assuming the absence of the gas phase. Let us begin with the Maxwell equations (3.2). The electric field to within terms of the order $\frac{1}{c}$ satisfies the equations obtained from (3.2), (3.3), (3.10):

$$
\begin{gathered}
\text { curl } \boldsymbol{E}=0, \\
\operatorname{div} \boldsymbol{D}=4 \pi \rho_{e}, \\
\frac{\partial \rho_{e}}{\partial t}+\operatorname{div} \boldsymbol{j}=0, \\
\boldsymbol{j}=\rho_{e}(\boldsymbol{v}+\boldsymbol{E} \Omega) .
\end{gathered}
$$

By virtue of Remark 2.1, in the absence of the gas phase the first law of thermodynamics (3.1) in electrohydrodynamical approximation becomes

$$
d \varepsilon_{0}=T d s-p d V+(\boldsymbol{E}, d \mathcal{P})+V\{(\boldsymbol{f}, d \boldsymbol{b})+\Phi d(\triangle w)\} .
$$

Here

$\varepsilon_{0}=e_{0}+V E_{0}$ is the internal energy,

$e_{0}=e_{0}(V, s, \mathcal{P})$ is the state equation,

$$
E_{0}=\left\{\frac{B}{8}\left(\frac{|\boldsymbol{b}|^{2}}{q^{2}}-1\right)^{2}+\frac{N}{2} \frac{(\triangle w)^{2}}{q^{2}}+\sigma q\left(\frac{1}{d_{3}}-1\right)\right\}
$$

is the volume density of "surface elastic energy" in the absence of the gas phase, $\sigma=$ const $\geqslant 0$ is the "surface tension", and

$$
\boldsymbol{f}=\frac{B}{2 q^{2}}\left(\frac{|\boldsymbol{b}|^{2}}{q^{2}}-1\right) \boldsymbol{b}+\frac{\sigma q}{b_{3}}\left(d_{1}, d_{2}, \frac{-d_{1}^{2}-d_{2}^{2}}{d_{3}}\right) .
$$

Let

$$
\begin{gathered}
\varepsilon_{0}=U+(\boldsymbol{E}, \mathcal{P}), \\
U=U_{0}+V E_{0},
\end{gathered}
$$

where $U_{0}=U_{0}(V, s, \boldsymbol{E})$ is the state equation.

In view of (4.4), the thermodynamic identity (4.2) becomes

$$
d U=T d s-p d V-(\mathcal{P}, d \boldsymbol{E})+V\{(\boldsymbol{f}, d \boldsymbol{b})+\Phi d(\triangle w)\} .
$$

It follows from (4.5) that

$$
\begin{gathered}
p=-\left(U_{0}\right)_{V}-E_{0}-V\left(E_{0}\right)_{V}, \\
\mathcal{P}=-\left(U_{0}\right)_{\boldsymbol{E}}=\left(-\left(U_{0}\right)_{E_{1}},-\left(U_{0}\right)_{E_{2}},-\left(U_{0}\right)_{E_{3}}\right) .
\end{gathered}
$$


We now begin to simplify system (3.6a) 3.6d). The main difficulty consists in the simplification of the law of impulse conservation (3.6c). In view of (3.4), we reduce it to (3.13e). By passing in (3.13e to electrohydrodynamical approximation (i.e., by dropping terms of the order $\frac{1}{c}$ ) we get

$$
\frac{d v_{i}}{d t}+V p_{x_{i}}=V \sum_{k=1}^{3}\left(\bar{\sigma}_{i k}\right)_{x_{k}}+\left(\mathcal{P}, \boldsymbol{E}_{x_{i}}\right)+\rho_{e} V E_{i}, i=1,2,3
$$

Here $\bar{\sigma}_{i k}=b_{i}\left(-f_{k}+\Phi_{x_{k}}\right)-\Phi B_{i k}, i, k=1,2,3$ (see (2.12)). Considering (3.6a), we rewrite (4.7) in the conservation form

$$
\left(\rho v_{i}\right)_{t}+p_{x_{i}}+\sum_{k=1}^{3}\left(\rho v_{i} v_{k}-\bar{\sigma}_{i k}\right)_{x_{k}}=\left(\boldsymbol{P}, \boldsymbol{E}_{x_{i}}\right)+\rho_{e} E_{i}, i=1,2,3 .
$$

Keeping in mind further applications (e.g., the study of the stability of shock waves), it is convenient to rewrite (4.8) as follows. In view of (4.1), we rewrite the right-hand side of (4.8):

$$
\begin{gathered}
\left(\boldsymbol{P}, \boldsymbol{E}_{x_{i}}\right)+\rho_{e} E_{i}=\frac{\left(4 \pi \boldsymbol{P}, \boldsymbol{E}_{x_{i}}\right)+E_{i} \operatorname{div} \boldsymbol{D}}{4 \pi} \\
=\frac{\left(\boldsymbol{D}, \boldsymbol{E}_{x_{i}}\right)+E_{i} \operatorname{div} \boldsymbol{D}-\left(\boldsymbol{E}, \boldsymbol{E}_{x_{i}}\right)}{4 \pi} \\
=\frac{(\boldsymbol{D}, \nabla) E_{i}+E_{i} \operatorname{div} \boldsymbol{D}}{4 \pi}-\frac{1}{8 \pi}\left(|\boldsymbol{E}|^{2}\right)_{x_{i}}=\frac{\operatorname{div}\left(E_{i} \boldsymbol{D}\right)}{4 \pi}-\frac{1}{8 \pi}\left(|\boldsymbol{E}|^{2}\right)_{x_{i}} .
\end{gathered}
$$

In view of (4.9), it follows from (4.8) that

$$
\left(\rho v_{i}\right)_{t}+\left\{p+\frac{|\boldsymbol{E}|^{2}}{8 \pi}\right\}_{x_{i}}+\sum_{k=1}^{3}\left(\rho v_{i} v_{k}-\Sigma_{i k}\right)_{x_{k}}=0, i=1,2,3,
$$

where

$$
\Sigma_{i k}=\bar{\sigma}_{i k}+\frac{E_{i} D_{k}}{4 \pi}
$$

Using the Umow-Pointing equation (3.5), we rewrite the law of energy conservation as follows:

$$
\left\{\rho\left(\varepsilon_{0}+\frac{|\boldsymbol{v}|^{2}}{2}\right)\right\}_{t}+\operatorname{div} \boldsymbol{Q}=(\boldsymbol{j}, \boldsymbol{E})+\left(\boldsymbol{E}, \boldsymbol{P}_{t}\right) .
$$

Here

$$
\begin{gathered}
\boldsymbol{Q}=\rho \boldsymbol{v}\left\{U+p V+\frac{|\boldsymbol{v}|^{2}}{2}\right\}+\boldsymbol{q}, \quad \boldsymbol{q}=\left(q_{1}, q_{2}, q_{3}\right), \\
\left.q_{k}=\left(f_{k}-\frac{\partial \Phi}{\partial x_{k}}\right)(\boldsymbol{b}, \boldsymbol{v})+\Phi \sum_{l=1}^{3}\left(v_{l} B_{k l}+b_{l} \frac{\partial v_{l}}{\partial x_{k}}\right), \quad k=1,2,3(\text { see (2.13) }) \text { and (2.14) }\right) .
\end{gathered}
$$

For a further simplification of the equations of layered structures in electrohydrodynamical approximation we consider the case when the state equation

$$
U_{0}=U_{0}(V, s, \boldsymbol{E})
$$

has the form

$$
U_{0}=\tilde{U}_{0}(V, s)-\frac{V}{2}(\boldsymbol{E}, \boldsymbol{E} \Psi)
$$


where $\Psi=\Psi^{*}$ is the constant matrix of dielectric sensitivity. It follows from (4.12) that

$$
\mathcal{P}=V \boldsymbol{E} \Psi,
$$

i.e.,

$$
\boldsymbol{P}=\boldsymbol{E} \Psi, \quad \boldsymbol{D}=\boldsymbol{E}\left(I_{3}+4 \pi \Psi\right)=\boldsymbol{E} \tilde{\Psi} .
$$

Let

$$
U=\tilde{U}-\frac{V}{2}(\boldsymbol{E}, \boldsymbol{E} \Psi), \quad \tilde{U}=\tilde{U}_{0}+V E_{0} .
$$

Then, the thermodynamic identity (4.5) is rewritten as

$$
d \tilde{U}=T d s-\tilde{p} d V+V\{(\boldsymbol{f}, d \boldsymbol{b})+\Phi d(\triangle w)\} .
$$

Here

$$
\tilde{p}=\left(p-\frac{1}{2}(\boldsymbol{E}, \boldsymbol{E} \Psi)\right)=-\left(\tilde{U}_{0}\right)_{V}-E_{0}-V\left(E_{0}\right)_{V} .
$$

Thus, taking into account (4.12), the law of energy conservation in electrohydrodynamical approximaton takes the form

$$
\left\{\rho\left(\tilde{U}+\frac{|\boldsymbol{v}|^{2}}{2}\right)\right\}_{t}+\operatorname{div} \boldsymbol{Q}=(\boldsymbol{j}, \boldsymbol{E}),
$$

where

$$
\boldsymbol{Q}=\rho \boldsymbol{v}\left\{\tilde{U}+\tilde{p} V+\frac{|\boldsymbol{v}|^{2}}{2}\right\}+\boldsymbol{q}
$$

and the vector $\boldsymbol{q}$ is described above. The system hydrodynamic equations of layered structures in electrohydrodynamical approximation is finally written as

$$
\begin{gathered}
\rho_{t}+\operatorname{div}(\rho \boldsymbol{v})=0 \\
\left(\rho v_{i}\right)_{t}+\left\{p+\frac{|\boldsymbol{E}|^{2}}{8 \pi}\right\}_{x_{i}}+\sum_{k=1}^{3}\left(\rho v_{i} v_{k}-\Sigma_{i k}\right)_{x_{k}}=0, \quad i=1,2,3 \\
(\rho s)_{t}+\operatorname{div}(\rho s \boldsymbol{v})=\frac{R}{T} \\
\left\{\rho\left(\tilde{U}+\frac{|\boldsymbol{v}|^{2}}{2}\right)\right\}_{t}+\operatorname{div} \boldsymbol{Q}=(\boldsymbol{j}, \boldsymbol{E}),
\end{gathered}
$$

where

$R=\rho_{e}(\boldsymbol{E} \Omega, \boldsymbol{E})$, and the components $\Sigma_{i k}$ of the stress tensor and the energy flux vector $\boldsymbol{Q}$ are described above.

Instead of system (4.16a)-4.16d), we have now the following nonconservative form:

$$
\begin{gathered}
\frac{d \rho}{d t}+\rho \operatorname{div} \boldsymbol{v}=0, \\
\frac{d w}{d t}=0, \\
\frac{d s}{d t}=\frac{R V}{T}, \\
\frac{d v_{i}}{d t}+V \tilde{p}_{x_{i}}=V \sum_{k=1}^{3}\left(\bar{\sigma}_{i k}\right)_{x_{k}}+\rho_{e} V E_{i}, \quad i=1,2,3 .
\end{gathered}
$$


Using relation (4.14), we can replace equation (4.17a) with the equation for $\tilde{p}$ :

$$
\frac{d \tilde{p}}{d t}+\rho c_{s}^{2} \operatorname{div} \boldsymbol{v}=\tilde{p}_{s} \frac{R V}{T}-\left(\boldsymbol{f}+V\left(E_{0}\right)_{V \boldsymbol{b}}, \frac{d \boldsymbol{b}}{d t}\right)-\left(1-2 \frac{q_{V}}{q} V\right) \Phi \frac{d(\triangle w)}{d t} .
$$

Here

$$
\begin{gathered}
c_{s}^{2}=\tilde{p}_{\rho}=-V^{2} \tilde{p}_{V}=V^{2}\left\{\left(\tilde{U}_{0}\right)_{V V}+2\left(E_{0}\right)_{V}+V\left(E_{0}\right)_{V V}\right\}, \\
\tilde{p}_{s}=-\left(\tilde{U}_{0}\right)_{V s}-\left(E_{0}\right)_{s}-V\left(E_{0}\right)_{V s},
\end{gathered}
$$

the terms $\left(E_{0}\right)_{V},\left(E_{0}\right)_{V V},\left(E_{0}\right)_{s},\left(E_{0}\right)_{V s},\left(E_{0}\right)_{V \boldsymbol{b}}$ are found from the formulas written above by assuming that $\boldsymbol{a} \equiv 0$, and the derivatives

$$
\frac{d \boldsymbol{b}}{d t}, \frac{d(\triangle w)}{d t}
$$

are defined from (2.10a), (2.10c).

REMARK 4.1. In equations (4.17d the influence of ponderomotive forces, i.e., the forces acting from the side of an electric field on a layered structure in which polarization is going on is carried out through the item $V \tilde{p}_{x_{i}}$.

REMARK 4.2. The law of energy conservation (4.16d) is a consequence of all the other consevation laws. Indeed, it follows from (4.13) that

$$
\begin{gathered}
\frac{\partial}{\partial t}\left\{\rho\left(\tilde{U}+\frac{|\boldsymbol{v}|^{2}}{2}\right)\right\}=\left(\tilde{U}+\tilde{p} V+\frac{|\boldsymbol{v}|^{2}}{2}\right) \frac{\partial \rho}{\partial t}+\left(\rho \boldsymbol{v}, \frac{\partial \boldsymbol{v}}{\partial t}\right) \\
+\rho T \frac{\partial s}{\partial t}+\left(\boldsymbol{f}, \frac{\partial \boldsymbol{b}}{\partial t}\right)+\Phi \frac{\partial(\triangle w)}{\partial t} .
\end{gathered}
$$

Substituting the derivatives

$$
\frac{\partial \rho}{\partial t}, \frac{\partial \boldsymbol{v}}{\partial t}, \frac{\partial s}{\partial t}, \frac{\partial \boldsymbol{b}}{\partial t}, \frac{\partial(\triangle w)}{\partial t}
$$

into the right-hand side of expression (4.20), we finally obtain the law of energy conservation 4.16d).

5. Linearization of the equations of an electrohydrodynamical model of a layered dielectric. The mathematical model (2.3), (4.1), 4.16a)-4.16d) (or (4.17a)(4.17d) ) obtained in the previous section has the following simple solution (it is called basic):

$$
\begin{gathered}
\rho=\hat{\rho}, s=\hat{s}, v_{1}=\hat{v}_{1}, v_{2}=\hat{v}_{2}, v_{3}=0, \\
w=\hat{w}=\hat{q} z, \\
\boldsymbol{E}=\hat{\boldsymbol{E}}=\left(\hat{E}_{1}, \hat{E}_{2}, \hat{E}_{3}\right), \rho_{e}=0 .
\end{gathered}
$$

Here

$\hat{\rho}, \hat{s}, \hat{v}_{1}, \hat{v}_{2}, \hat{E}_{1}, \hat{E}_{2}, \hat{E}_{3}$ are some constants,

$$
\hat{q}=q(\hat{V}, \hat{s}), \hat{V}=\frac{1}{\hat{\rho}} .
$$

Linearizing system (4.17a)-(4.17d) about the basic solution (5.1), one gets (small perturbations are denoted by the same letters)

$$
\begin{gathered}
\hat{L} w+\hat{q} v_{3}=0, \\
\hat{L} s=\rho_{e} \hat{a},
\end{gathered}
$$




$$
\begin{gathered}
\hat{L} v_{i}+\hat{V} \tilde{p}_{x_{i}}=\hat{a}_{i} \rho_{e}, i=1,2, \\
\hat{L} v_{3}+\hat{V} \hat{\beta} \tilde{p}_{x_{3}}=\frac{N}{\hat{\rho} \hat{q}} \triangle^{2} w-\frac{B}{\hat{\rho} \hat{q}} \hat{\lambda}^{2} \frac{\partial^{2} w}{\partial x_{3}^{2}}-\hat{V} \sigma \triangle_{\perp} w+\Sigma \frac{\partial s}{\partial x_{3}}+\hat{a}_{3} \rho_{e}, \\
\hat{L} \tilde{p}+\hat{\rho} \hat{c}_{s}^{2} \operatorname{div} \boldsymbol{v}-B \hat{\gamma} \frac{\partial v_{3}}{\partial x_{3}}=\hat{\tilde{p}}_{s} \hat{a} \rho_{e},
\end{gathered}
$$

where

$$
\begin{gathered}
\hat{L}=\frac{\partial}{\partial t}+\hat{v}_{1} \frac{\partial}{\partial x_{1}}+\hat{v}_{2} \frac{\partial}{\partial x_{2}}, \\
\hat{a}=\frac{(\hat{\boldsymbol{E}} \hat{\Omega}, \hat{\boldsymbol{E}})}{\hat{\rho} \hat{T}}=\frac{\omega_{\perp}\left(\hat{E}_{1}^{2}+\hat{E}_{2}^{2}\right)+\omega_{\|} \hat{E}_{3}^{2}}{\hat{\rho} \hat{T}}(\text { see statement (3.11) }),
\end{gathered}
$$

$\omega_{\perp}, \omega_{\|} \geqslant 0$ are some constants,

$$
\begin{gathered}
\hat{a}_{i}=\hat{V} \hat{E}_{i}, i=1,2,3, \quad \hat{\beta}=1-\hat{B} \hat{\gamma}, \quad \hat{B}=\frac{B}{\hat{\rho} \hat{c}_{s}^{2}}, \quad \hat{\gamma}=\gamma(\hat{\rho}, \hat{s}), \\
\gamma=\frac{\rho}{q} \frac{\partial q}{\partial \rho}, \quad \triangle_{\perp}=\frac{\partial^{2}}{\partial x_{1}^{2}}+\frac{\partial^{2}}{\partial x_{2}^{2}}, \quad \triangle=\triangle_{\perp}+\frac{\partial^{2}}{\partial x_{3}^{2}}, \\
\Sigma=\hat{B}\left\{\hat{V} \hat{\gamma}\left(\hat{\tilde{U}}_{0}\right)_{V_{S}}+\hat{\rho} \hat{\not} \hat{\lambda}^{2} \hat{c}_{s}^{2}\right\} \\
\hat{æ}=æ(\hat{\rho}, \hat{s}), \quad \rho æ=\frac{1}{q} \frac{\partial q}{\partial s}, \quad \hat{\lambda}^{2}=1-\hat{B} \hat{\gamma}^{2}, \\
\left(\hat{\tilde{U}}_{0}\right)_{V_{S}}=\left(\tilde{U}_{0}\right)_{V_{S}}(\hat{V}, \hat{s}), \\
\hat{\rho}^{2} \hat{c}_{s}^{2}=\left\{\left(\hat{\tilde{U}}_{0}\right)_{V V}+B \hat{\rho} \hat{\gamma}^{2}\right\} \quad(\text { see formula (4.19) }), \\
\left(\hat{\tilde{U}}_{0}\right)_{V V}=\left(\tilde{U}_{0}\right)_{V V}(\hat{V}, \hat{s}), \quad \tilde{p}=p-(\hat{\boldsymbol{E}} \Psi, \boldsymbol{E}) .
\end{gathered}
$$

Note that while obtaining the linearized system (5.2a - (5.2e), we used the following expression, which is a linear analogue of (4.14):

$$
\tilde{p}=-\hat{\rho}^{2} \hat{c}_{s}^{2} V+\hat{\tilde{p}}_{s} s-\frac{B \hat{\gamma}}{\hat{q}} \frac{\partial w}{\partial x_{3}} .
$$

Here $\hat{\tilde{p}}_{s}=-\left(\hat{\tilde{U}}_{0}\right)_{V_{S}}+B \hat{\gamma} \hat{\rho} \hat{\not}$. Moreover, it follows from (5.3) that

$$
\hat{\lambda}^{2}=\frac{\hat{c}^{2}}{\hat{c}_{s}^{2}}=1-\hat{B} \hat{\gamma}^{2} .
$$

Here $\hat{c}^{2}=\hat{V}^{2}\left(\hat{\tilde{U}}_{0}\right)_{V V}$.

The linearization of the Maxwell equations (4.1) gives us the relations

$$
\begin{gathered}
\operatorname{curl} \boldsymbol{E}=0, \\
\operatorname{div}(\boldsymbol{E} \tilde{\Psi})=4 \pi \rho_{e}, \\
\frac{\partial \rho_{e}}{\partial t}+((\hat{\boldsymbol{v}}+\hat{\boldsymbol{E}} \hat{\Omega}), \nabla) \rho_{e}=0 .
\end{gathered}
$$

Here $\nabla=\left(\frac{\partial}{\partial x_{1}}, \frac{\partial}{\partial x_{2}}, \frac{\partial}{\partial x_{3}}\right)$. While deriving equations (5.5a)-(5.5c), we used the relation $\boldsymbol{D}=\boldsymbol{E} \tilde{\Psi}$. Moreover (see (3.11)),

$$
\hat{\boldsymbol{E}} \hat{\Omega}=\left(\omega_{\perp} \hat{E}_{1}, \omega_{\perp} \hat{E}_{2}, \omega_{\|} \hat{E}_{3}\right)=\hat{\boldsymbol{\xi}} .
$$


Again coming back to the linear system (5.2a)-(5.2e), it should be noted that it is more convenient to use its dimensionless version. For this purpose, we introduce new dependent and independent variables:

$$
\begin{gathered}
x_{i}^{\prime}=\frac{x_{i}}{z_{0}}, i=1,2,3, \\
w^{\prime}=\frac{w}{\hat{q} z_{0}}, s^{\prime}=\frac{s}{\hat{s}}, \\
v_{i}^{\prime}=\frac{v_{i}}{\hat{c}_{s}}, i=1,2,3, \\
t^{\prime}=\frac{\hat{c}_{s}}{z_{0}} t, p^{\prime}=\frac{p}{\hat{\rho} \hat{c}_{s}^{2}}, \rho^{\prime}=\frac{\rho}{\hat{\rho}}, V^{\prime}=\hat{\rho} V, \\
\rho_{e}^{\prime}=\rho_{e} \frac{z_{0} \hat{a}}{\hat{s} \hat{c}_{s}}=\rho_{e} z_{0} \hat{A}, \tilde{p}^{\prime}=p^{\prime}-\left(\hat{\boldsymbol{A}} \Psi, \boldsymbol{E}^{\prime}\right), \boldsymbol{E}^{\prime}=\hat{A} \boldsymbol{E} .
\end{gathered}
$$

Here $z_{0}>0$ characterizes the thickness of a film, and the vector $\hat{\boldsymbol{A}}=\left(\hat{A}_{1}, \hat{A}_{2}, \hat{A}_{3}\right)$ is described below. Then, system (5.2a) $-(5.2 \mathrm{e})$ becomes (below we drop the primes):

$$
\begin{gathered}
L w+v_{3}=0, \\
L s=\rho_{e}, \\
L v_{i}+\tilde{p}_{x_{i}}=\hat{A}_{i} \rho_{e}, i=1,2, \\
L v_{3}+\hat{\beta} \tilde{p}_{x_{3}}=\hat{N} \triangle^{2} w-\hat{B} \hat{\lambda}^{2} \frac{\partial^{2} w}{\partial x_{3}^{2}}-\hat{\sigma} \triangle_{\perp} w+\hat{\Sigma} \frac{\partial s}{\partial x_{3}}+\hat{A}_{3} \rho_{e}, \\
L \tilde{p}+\operatorname{div}_{\perp} \boldsymbol{v}+\hat{\beta} \frac{\partial v_{3}}{\partial x_{3}}=R_{1} \rho_{e}
\end{gathered}
$$

where

$$
\begin{gathered}
L=\frac{\partial}{\partial t}+M_{1} \frac{\partial}{\partial x_{1}}+M_{2} \frac{\partial}{\partial x_{2}}, M_{i}=\frac{\hat{v}_{i}}{\hat{c}_{s}}, i=1,2, \\
\hat{A}_{i}=\frac{\hat{a}_{i}}{\hat{A} \hat{c}_{s}^{2}}, i=1,2,3, \\
\hat{N}=\frac{N}{\hat{\rho} \hat{c}_{s}^{2} z_{0}^{2}}, \hat{\sigma}=\frac{\sigma \hat{q}}{\hat{\rho} \hat{c}_{s}^{2}}, \hat{\Sigma}=\frac{\Sigma \hat{s}}{\hat{c}_{s}^{2}}, \\
\operatorname{div}_{\perp} \boldsymbol{v}=\frac{\partial v_{1}}{\partial x_{1}}+\frac{\partial v_{2}}{\partial x_{2}}, R_{1}=\frac{\hat{s}\left\{B \hat{\gamma} \hat{\rho} \hat{\not}-\left(\hat{\tilde{U}}_{0}\right)_{V_{s}}\right\}}{\hat{\rho} \hat{c}_{s}^{2}} .
\end{gathered}
$$

Relation (5.4) in a dimensionless form looks as follows:

$$
\tilde{p}=-V+R_{1} s-\hat{B} \hat{\gamma} \frac{\partial w}{\partial x_{3}} .
$$

The linearized Maxwell equations (5.5a) $-(5.5 \mathrm{C}$ ) in a dimensionless form are:

$$
\begin{gathered}
\operatorname{curl} \boldsymbol{E}=0, \\
\operatorname{div}(\boldsymbol{E} \tilde{\Psi})=4 \pi \rho_{e}, \\
L \rho_{e}+\left(\frac{\hat{\boldsymbol{\xi}}}{\hat{c}_{s}}, \nabla\right) \rho_{e}=0 .
\end{gathered}
$$


At the end of this section we get some consequences of equations (5.7a) $-(5.7 \mathrm{e})$. Let us act on equation (5.7e) by the operator $L$. Then, in view of (5.7a) and (5.7b), one gets

$$
L^{2} v_{3}-\hat{B} \hat{\lambda}^{2} \frac{\partial^{2} v_{3}}{\partial x_{3}^{2}}-\hat{\sigma} \triangle_{\perp} v_{3}+\hat{N} \triangle^{2} v_{3}+\hat{\beta} \frac{\partial(L \tilde{p})}{\partial x_{3}}=\hat{\Sigma} \frac{\partial \rho_{e}}{\partial x_{3}}+\hat{A}_{3} L \rho_{e} .
$$

It follows from (5.7c), (5.7d $)$ that

$$
L\left(\operatorname{div}_{\perp} \boldsymbol{v}\right)+\triangle_{\perp} \tilde{p}=\sum_{i=1}^{2} \hat{A}_{i} \frac{\partial \rho_{e}}{\partial x_{i}} .
$$

By virtue of this relation, equation (5.7e) implies

$$
L^{2} \tilde{p}-\triangle_{\perp} \tilde{p}+\hat{\beta} \frac{\partial\left(L v_{3}\right)}{\partial x_{3}}=R_{1} L \rho_{e}-\sum_{i=1}^{2} \hat{A}_{i} \frac{\partial \rho_{e}}{\partial x_{i}} .
$$

REMARK 5.1. It was shown above that from system (5.7a)-(5.7e), (5.9a)-(5.9c) one can obtain a closed subsystem for the functions $v_{3}, \tilde{p}, \rho_{e}$. We now consider the Cauchy problem for this subsystem. For this purpose, we add to it the initial data

$$
\begin{gathered}
\left.v_{3}\right|_{t=0}=v_{30}(\boldsymbol{x}),\left.\quad \frac{\partial v_{3}}{\partial t}\right|_{t=0}=v_{31}(\boldsymbol{x}), \\
\left.\tilde{p}\right|_{t=0}=\tilde{p}_{0}(\boldsymbol{x}),\left.\quad \frac{\partial \tilde{p}}{\partial t}\right|_{t=0}=\tilde{p}_{1}(\boldsymbol{x}), \\
\left.\rho_{e}\right|_{t=0}=\rho_{e 0}(\boldsymbol{x}) .
\end{gathered}
$$

We note also that, performing in (5.9c), (5.10), (5.11) the Galilean transform (below we drop the primes)

$$
\begin{gathered}
t^{\prime}=t, \\
x_{j}^{\prime}=x_{j}-M_{j} t, i=1,2,
\end{gathered}
$$

we rewrite the closed subsystem as follows:

$$
\begin{gathered}
\tau^{2} v_{3}-\hat{B} \hat{\lambda}^{2} \xi_{3}^{2} v_{3}-\hat{\sigma} \triangle_{\perp} v_{3}+\hat{N} \triangle^{2} v_{3}+\hat{\beta} \tau \xi_{3} \tilde{p}-\hat{\Sigma} \xi_{3} \rho_{e}-\hat{A}_{3} \tau \rho_{e}=0 \\
\tau^{2} \tilde{p}-\triangle_{\perp} \tilde{p}+\hat{\beta} \tau \xi_{3} v_{3}-R_{1} \tau \rho_{e}+\sum_{j=1}^{2} \hat{A}_{j} \xi_{j} \rho_{e}=0 \\
\tau \rho_{e}+(\hat{\Lambda}, \nabla) \rho_{e}=0 .
\end{gathered}
$$

Here

$$
\begin{gathered}
\tau=\frac{\partial}{\partial t}, \xi_{j}=\frac{\partial}{\partial x_{j}}, j=1,2,3, \\
\hat{\boldsymbol{\Lambda}}=\frac{\hat{\boldsymbol{\xi}}}{\hat{c}_{s}} .
\end{gathered}
$$

Let us get an a priori estimate for the Cauchy problem (5.14a)-(5.14c), (5.12), which implies well-posedness. We have the relations

$$
\begin{gathered}
2 \tau v_{3} \tau^{2} v_{3}=\tau\left(\tau v_{3}\right)^{2}, \\
2 \tau v_{3} \xi_{j}^{2} v_{3}=2 \xi_{j}\left(\tau v_{3} \xi_{j} v_{3}\right)-\tau\left(\xi_{j} v_{3}\right)^{2}, j=1,2,3, \\
2 \tau v_{3} \xi_{j}^{2} \triangle v_{3}=2 \xi_{j}\left(\tau v_{3} \xi_{j} \triangle v_{3}\right)-2 \xi_{j} \triangle v_{3} \tau \xi_{j} v_{3}, j=1,2,3,
\end{gathered}
$$




$$
2 \xi_{j} \triangle v_{3} \tau \xi_{j} v_{3}=2 \sum_{k=1}^{3} \tau \xi_{j} v_{3} \xi_{j} \xi_{k}^{2} v_{3}=2 \sum_{k=1}^{3} \xi_{k}\left(\xi_{j} \xi_{k} v_{3} \tau \xi_{j} v_{3}\right)-\sum_{k=1}^{3} \tau\left(\xi_{j} \xi_{k} v_{3}\right)^{2}, j=1,2,3 .
$$

From equation (5.14c) we easily obtain

$$
\begin{gathered}
\tau\left(\tau \rho_{e}\right)+(\hat{\boldsymbol{\Lambda}}, \nabla)\left(\tau \rho_{e}\right)=0, \\
\tau\left(\xi_{j} \rho_{e}\right)+(\hat{\boldsymbol{\Lambda}}, \nabla)\left(\xi_{j} \rho_{e}\right)=0, j=1,2,3 .
\end{gathered}
$$

Let us multiply equation (5.14a) by $2 \tau v_{3}$, equation (5.14b) by $2 \tau \tilde{p}$, equation (5.14c) by $2 \rho_{e}$, equation (5.15) by $2 \tau \rho_{e}$, and relations (5.16) by $2 \xi_{j} \rho_{e}, j=1,2,3$, respectively. Then, by summing the obtained expressions one gets

$$
\begin{array}{r}
\tau\left\{\left(\tau v_{3}\right)^{2}+\hat{B} \hat{\lambda}^{2}\left(\xi_{3} v_{3}\right)^{2}+\hat{\sigma}\left(\xi_{1} v_{3}\right)^{2}+\hat{\sigma}\left(\xi_{2} v_{3}\right)^{2}+\hat{N} \sum_{j=1}^{3} \sum_{k=1}^{3}\left(\xi_{j} \xi_{k} v_{3}\right)^{2}\right. \\
\left.+(\tau \tilde{p})^{2}+\left(\xi_{1} \tilde{p}\right)^{2}+\left(\xi_{2} \tilde{p}\right)^{2}+\rho_{e}^{2}+\left(\tau \rho_{e}\right)^{2}+\sum_{j=1}^{3}\left(\xi_{j} \rho_{e}\right)^{2}\right\} \\
+2 \hat{\beta} \xi_{3}\left(\tau \tilde{p} \tau v_{3}\right)-2 \hat{B} \hat{\lambda}^{2} \xi_{3}\left(\tau v_{3} \xi_{3} v_{3}\right)-2 \hat{\sigma} \sum_{j=1}^{2} \xi_{j}\left(\tau v_{3} \xi_{j} v_{3}\right) \\
+2 \hat{N} \sum_{j=1}^{3} \xi_{j}\left(\tau v_{3} \xi_{j} \triangle v_{3}\right)-2 \hat{N} \sum_{j=1}^{3} \sum_{k=1}^{3} \xi_{k}\left(\xi_{j} \xi_{k} v_{3} \tau \xi_{j} v_{3}\right) \\
-2 \sum_{j=1}^{2} \xi_{j}\left(\tau \tilde{p} \xi_{j} \tilde{p}\right)+(\hat{\boldsymbol{\Lambda}}, \nabla)\left(\rho_{e}\right)^{2}+(\hat{\boldsymbol{\Lambda}}, \nabla)\left(\tau \rho_{e}\right)^{2}+\sum_{j=1}^{3}(\hat{\boldsymbol{\Lambda}}, \nabla)\left(\xi_{j} \rho_{e}\right)^{2} \\
=2 \hat{A}_{3} \tau v_{3} \tau \rho_{e}+2 \hat{\Sigma} \tau v_{3} \xi_{3} \rho_{e}+2 R_{1} \tau \tilde{p} \tau \rho_{e}-2 \sum_{j=1}^{2} \hat{A}_{j} \tau \tilde{p} \xi_{j} \rho_{e} .
\end{array}
$$

Integrating equality (5.17) over $\mathbb{R}^{3}$ and assuming that the functions $v_{3}, \tilde{p}, \rho_{e}$ and their derivatives tend to zero as $\left|x_{j}\right| \rightarrow \infty, j=1,2,3$, we finally get

$$
\frac{d}{d t} J(t) \leq \tilde{M} J(t)
$$

where

$$
\begin{aligned}
J(t)=\int_{\mathbb{R}^{3}}\{ & \left(\tau v_{3}\right)^{2}+\hat{B} \hat{\lambda}^{2}\left(\xi_{3} v_{3}\right)^{2}+\hat{\sigma} \sum_{j=1}^{2}\left(\xi_{j} v_{3}\right)^{2}+\hat{N} \sum_{j=1}^{3} \sum_{k=1}^{3}\left(\xi_{j} \xi_{k} v_{3}\right)^{2} \\
& \left.+(\tau \tilde{p})^{2}+\sum_{j=1}^{2}\left(\xi_{j} \tilde{p}\right)^{2}+\rho_{e}^{2}+\left(\tau \rho_{e}\right)^{2}+\sum_{j=1}^{3}\left(\xi_{j} \rho_{e}\right)^{2}\right\} d x_{1} d x_{2} d x_{3},
\end{aligned}
$$

$\tilde{M}>0$ is a constant.

From inequality (5.18) we get the desired a priori estimate

$$
J(t) \leq e^{\tilde{M} t} J(0)
$$

where $J(0)$ in (5.19) is expressed through the initial data (5.12). 
6. Strong discontinuities in a layered dielectric. By standard arguments (see [3, 6, 9, 12]) we get the following Rankine-Hugoniot conditions for equations (4.17a)(4.17d), (2.3), (4.1):

$$
\begin{gathered}
{\left[\rho\left(v_{n}-\mathcal{D}_{n}\right)\right]=[\mathrm{J}]=0,} \\
\mathrm{~J}[w]=0, \\
\mathrm{~J}\left[v_{n}\right]+[\Pi]=0, \\
\mathrm{~J}\left[v_{\tau}\right]-[(\boldsymbol{C}, \boldsymbol{\tau})]=0, \\
\mathrm{~J}\left[\tilde{U}+\frac{|\boldsymbol{v}|^{2}}{2}\right]+[G]=0, \\
{\left[j_{n}\right]=\frac{\partial \omega}{\partial t},} \\
{\left[D_{n}\right]=-4 \pi \omega,} \\
{\left[E_{\tau}\right]=0 .}
\end{gathered}
$$

Here

$\tilde{f}=(f(t, y, z)-x)=\left(f\left(t, x_{2}, x_{3}\right)-x_{1}\right)$ is a surface of strong discontinuity,

$\boldsymbol{n}=\left(n_{1}, n_{2}, n_{3}\right)=\frac{1}{\sqrt{1+f_{x_{2}}^{2}+f_{x_{3}}^{2}}}\left(-1, f_{x_{2}}, f_{x_{3}}\right)$ is the unit normal to the discontinuity surface,

$[j]=\left(j-j_{\infty}\right)$ denotes the jump of $j$ on the discontinuity surface, with $j_{\infty}$ the value of $j$ from the left of the discontinuity (i.e., when $\left.\left(f\left(t, x_{2}, x_{3}\right)-x_{1}\right) \rightarrow+0\right)$,

$v_{n}=(\boldsymbol{v}, \boldsymbol{n}), \quad v_{\tau}=(\boldsymbol{v}, \boldsymbol{\tau})$,

$\mathcal{D}_{n}=-\frac{f_{t}}{\sqrt{1+f_{x_{2}}^{2}+f_{x_{3}}^{2}}}$ is the normal component of the discontinuity speed, $\boldsymbol{\tau}=\left\{\begin{array}{l}\left(f_{x_{2}}, 1,0\right) \\ \left(f_{x_{3}}, 0,1\right)\end{array}\right.$ are the vectors orthogonal to the normal $\boldsymbol{n}$ and tangent to the discontinuity surface,

$$
\begin{aligned}
& \Pi=p+\frac{|\boldsymbol{E}|^{2}}{8 \pi}-(\boldsymbol{C}, \boldsymbol{n})=p+\frac{|\boldsymbol{E}|^{2}}{8 \pi}+b_{n}\left(f_{n}-\frac{\partial \Phi}{\partial n}\right)+\Phi(\boldsymbol{n} \mathcal{B}, \boldsymbol{n})-\frac{E_{n} D_{n}}{4 \pi}, \\
& \boldsymbol{C}=\left(\left(\boldsymbol{\Sigma}_{1}, \boldsymbol{n}\right),\left(\boldsymbol{\Sigma}_{2}, \boldsymbol{n}\right),\left(\boldsymbol{\Sigma}_{3}, \boldsymbol{n}\right)\right), \boldsymbol{\Sigma}_{i}=\left(\Sigma_{i 1}, \Sigma_{i 2}, \Sigma_{i 3}\right), i=1,2,3, \\
& \mathcal{B}=\left(B_{i k}\right)=\mathcal{B}^{*}, i, k=1,2,3, b_{n}=(\boldsymbol{b}, \boldsymbol{n}), f_{n}=(\boldsymbol{f}, \boldsymbol{n}), \\
& E_{n}=(\boldsymbol{E}, \boldsymbol{n}), D_{n}=(\boldsymbol{D}, \boldsymbol{n})=(\boldsymbol{E} \tilde{\Psi}, \boldsymbol{n}), \frac{\partial \Phi}{\partial n}=(\boldsymbol{n}, \nabla \Phi), \\
& -(\boldsymbol{C}, \boldsymbol{\tau})=b_{\tau}\left(f_{n}-\frac{\partial \Phi}{\partial n}\right)+\Phi(\boldsymbol{n \mathcal { B }}, \boldsymbol{\tau})-\frac{E_{\tau} D_{n}}{4 \pi}, b_{\tau}=(\boldsymbol{b}, \boldsymbol{\tau}), E_{\tau}=(\boldsymbol{E}, \boldsymbol{\tau}), \\
& G=\tilde{p} v_{n}+\Phi\left(\boldsymbol{b}, \frac{\partial \boldsymbol{v}}{\partial n}\right)+\Phi(\boldsymbol{n \mathcal { B }}, \boldsymbol{v}), \\
& v_{b}=(\boldsymbol{v}, \boldsymbol{b}), \frac{\partial \boldsymbol{v}}{\partial n}=(\boldsymbol{n}, \nabla) \boldsymbol{v}, j_{n}=(\boldsymbol{j}, \boldsymbol{n})=\rho_{e}((\boldsymbol{v}+\boldsymbol{E} \Omega), \boldsymbol{n})=\rho_{e} v_{n}+\rho_{e}(\boldsymbol{E} \Omega, \boldsymbol{n}) .
\end{aligned}
$$

While getting the jump conditions for system (4.1) we assumed the existence of a surface charge $\omega=\omega\left(t, \boldsymbol{x}^{\prime}\right)$ (where $\boldsymbol{x}^{\prime}=\left(x_{2}, x_{3}\right)$ ) and the absence of a surface current on the discontinuity surface (see [3]). 
We are interested in strong discontinuities of the type of shock wave (see [13), i.e. when

$$
[\rho] \neq 0, \mathrm{~J} \neq 0 .
$$

We consider a stationary flow of a layered dielectric with a surface of shock wave. Such a flow is described, for example, by a piecewise constant solution

$$
\begin{gathered}
\boldsymbol{v}=\left(\hat{v}_{1}, \hat{v}_{2}, 0\right), \rho=\hat{\rho}, s=\hat{s}, \\
w=\hat{q} z, \hat{q}=q(\hat{V}, \hat{s}), \hat{V}=\frac{1}{\hat{\rho}}, \\
\hat{\boldsymbol{E}}=\left(\hat{E}_{1}, \hat{E}_{2}, \hat{E}_{3}\right), \rho_{e}=0 \quad \text { with } x_{1}>0, \\
\boldsymbol{v}=\left(\hat{v}_{1 \infty}, \hat{v}_{2 \infty}, 0\right), \rho=\hat{\rho}_{\infty}, s=\hat{s}_{\infty}, \\
w=\hat{q}_{\infty} z, \hat{q}_{\infty}=q\left(\hat{V}_{\infty}, \hat{s}_{\infty}\right), \hat{V}_{\infty}=\frac{1}{\hat{\rho}_{\infty}}, \\
\hat{\boldsymbol{E}}=\left(\hat{E}_{1 \infty}, \hat{E}_{2 \infty}, \hat{E}_{3 \infty}\right), \rho_{e \infty}=0 \quad \text { with } x_{1}<0,
\end{gathered}
$$

where the constants

$$
\hat{v}_{1,2}, \hat{\rho}, \hat{s}, \hat{q}, \hat{q}_{\infty}, \hat{v}_{1,2 \infty}, \hat{\rho}_{\infty}, \hat{s}_{\infty}, \hat{E}_{1,2,3}, \hat{E}_{1,2,3 \infty}
$$

are connected by the jump conditions (6.1a) - (6.1h) on the stationary shock wave (with the equation $\left.x_{1}=0\right)$ :

$$
\begin{aligned}
& \hat{\rho} \hat{v}_{1}=\hat{\rho}_{\infty} \hat{v}_{1 \infty}=\hat{\mathrm{J}}, \hat{\mathrm{J}} \neq 0,[\hat{\rho}] \neq 0, \\
& \hat{q}=\hat{q}_{\infty}, \\
& \hat{\rho} \hat{v}_{1}^{2}+\hat{p}+\frac{|\hat{\boldsymbol{E}}|^{2}}{8 \pi}-\frac{\hat{E}_{1}\left(\sum_{i=1}^{3} \hat{E}_{i} \tilde{\Psi}_{i 1}\right)}{4 \pi} \\
& =\hat{\rho}_{\infty} \hat{v}_{1 \infty}^{2}+\hat{p}_{\infty}+\frac{\left|\hat{\boldsymbol{E}}_{\infty}\right|^{2}}{8 \pi}-\frac{\hat{E}_{1 \infty}\left(\sum_{i=1}^{3} \hat{E}_{i \infty} \tilde{\Psi}_{i 1 \infty}\right)}{4 \pi}, \\
& \hat{\mathrm{J}}\left[\hat{v}_{2}\right]-\left[\frac{\hat{E}_{2}\left(\sum_{i=1}^{3} \hat{E}_{i} \tilde{\Psi}_{i 1}\right)}{4 \pi}\right]=0, \\
& {\left[\frac{\hat{E}_{3}\left(\sum_{i=1}^{3} \hat{E}_{i} \tilde{\Psi}_{i 1}\right)}{4 \pi}\right]=0} \\
& \hat{\tilde{p}} \hat{V}+\hat{\tilde{U}}_{0}+\frac{|\hat{\boldsymbol{v}}|^{2}}{2}=\hat{\tilde{p}}_{\infty} \hat{V}_{\infty}+\left(\hat{\tilde{U}}_{0}\right)_{\infty}+\frac{\left|\hat{\boldsymbol{v}}_{\infty}\right|^{2}}{2} \\
& {\left[\sum_{i=1}^{3} \hat{E}_{i} \tilde{\Psi}_{i 1}\right]=4 \pi \hat{\omega}} \\
& {\left[\hat{E}_{k}\right]=0, k=2,3 \text {. }}
\end{aligned}
$$


Here

$$
\begin{gathered}
\hat{\tilde{p}}=\hat{p}-\frac{1}{2}(\hat{\boldsymbol{E}} \Psi, \hat{\boldsymbol{E}})=-\left(\tilde{U}_{0}\right)_{V}(\hat{V}, \hat{s}), \hat{\tilde{p}}_{\infty}=\hat{p}_{\infty}-\frac{1}{2}\left(\hat{\boldsymbol{E}}_{\infty} \Psi_{\infty}, \hat{\boldsymbol{E}}_{\infty}\right)=-\left(\tilde{U}_{0}\right)_{V}\left(\hat{V}_{\infty}, \hat{s}_{\infty}\right), \\
\left(\hat{\tilde{U}}_{0}\right)_{\infty}=\tilde{U}_{0}\left(\hat{V}_{\infty}, \hat{s}_{\infty}\right), \hat{\tilde{U}}_{0}=\tilde{U}_{0}(\hat{V}, \hat{s}) .
\end{gathered}
$$

To simplify relations (6.3) we assume that

$$
\hat{E}_{2,3}, \hat{E}_{2,3 \infty}=0 .
$$

As a result, instead of (6.3) we have

$$
\begin{gathered}
\hat{\rho} \hat{v}_{1}=\hat{\rho}_{\infty} \hat{v}_{1 \infty} \\
\hat{q}=\hat{q}_{\infty} \\
\hat{\rho} \hat{v}_{1}^{2}+\hat{\tilde{p}}+\hat{r} \hat{E}_{1}^{2}=\hat{\rho}_{\infty} \hat{v}_{1 \infty}^{2}+\hat{\tilde{p}}_{\infty}+\hat{r}_{\infty} \hat{E}_{1 \infty}^{2} \\
\hat{v}_{2}=\hat{v}_{2 \infty} \\
\hat{\tilde{p}} \hat{V}+\hat{\tilde{U}}_{0}+\frac{\hat{v}_{1}^{2}}{2}=\hat{\tilde{p}}_{\infty} \hat{V}_{\infty}+\left(\hat{\tilde{U}}_{0}\right)_{\infty}+\frac{\hat{v}_{1 \infty}^{2}}{2} \\
\hat{E}_{1} \tilde{\Psi}_{11}=\hat{E}_{1 \infty} \tilde{\Psi}_{11 \infty}+4 \pi \hat{\omega}
\end{gathered}
$$

where

$$
\begin{gathered}
\hat{r}=\frac{1}{2} \tilde{\Psi}_{11}+\frac{1}{8 \pi}-\frac{\tilde{\Psi}_{11}}{4 \pi}, \\
\hat{r}_{\infty}=\frac{1}{2} \tilde{\Psi}_{11 \infty}+\frac{1}{8 \pi}-\frac{\tilde{\Psi}_{11 \infty}}{4 \pi} .
\end{gathered}
$$

Let us now formulate a linearized stability problem for shock waves. We linearize the system of conservation laws (4.17a) -(4.17d) and the jump conditions (6.1a) (6.1h) about the piecewise constant solution (6.2) and reduce the result to a dimensionless form by introducing the following new independent and dependent variables:

$$
\begin{gathered}
x_{i}^{\prime}=\frac{x_{i}}{z_{0}}, i=1,2,3 \\
w^{\prime}=\frac{w}{\hat{q} z_{0}}, s^{\prime}=\frac{s}{\hat{s}} \\
v_{i}^{\prime}=\frac{v_{i}}{\hat{c}_{s}}, i=1,2,3 \\
t_{e}^{\prime}=\frac{\hat{c}_{s}}{z_{0}} t, p^{\prime}=\frac{p}{\hat{\rho} \hat{c}_{s}^{2}}, \rho^{\prime}=\frac{\rho}{\hat{\rho}}, V^{\prime}=\hat{\rho} V, \omega^{\prime}=\frac{\omega}{\hat{\omega}}, \\
\hat{\rho_{0} \hat{s} \hat{c}_{s}}=\rho_{e} z_{0} \hat{A}, \tilde{p}^{\prime}=p^{\prime}-\left(\hat{\mathbf{A}} \Psi, \boldsymbol{E}^{\prime}\right), \boldsymbol{E}^{\prime}=\hat{A} \boldsymbol{E}, f^{\prime}=\frac{f}{z_{0}} \\
\hat{\mathbf{A}}^{\prime}=\left(A_{1}, A_{2}, A_{3}\right), \hat{A}_{i}=\frac{\hat{a}_{i}}{\hat{A} \hat{c}_{s}^{2}}, \hat{a}_{i}=\hat{V} \hat{E}_{i}, i=1,2,3, \\
w_{\infty}^{\prime}=\frac{w_{\infty}}{\hat{q}_{\infty} z_{0}}, s_{\infty}^{\prime}=\frac{s_{\infty}}{\hat{s}_{\infty}}, \\
v_{i \infty}^{\prime}=\frac{v_{i \infty}}{\hat{c}_{s \infty}}, i=1,2,3 \\
p_{\infty} \\
p_{\infty}^{\prime}=\frac{\rho_{\infty}^{\prime}}{\hat{\rho}_{\infty} \hat{c}_{s \infty}^{2}}=\frac{\rho_{\infty}}{\hat{\rho}_{\infty}}, V_{\infty}^{\prime}=\hat{\rho}_{\infty} V_{\infty}
\end{gathered}
$$




$$
\begin{gathered}
\rho_{e \infty}^{\prime}=\rho_{e \infty} \frac{z_{0} \hat{a}_{\infty}}{\hat{s}_{\infty} \hat{c}_{s \infty}}=\rho_{e \infty} z_{0} \hat{A}_{\infty}, \tilde{p}_{\infty}^{\prime}=p_{\infty}^{\prime}-\left(\hat{\mathbf{A}}_{\infty} \Psi_{\infty}, \boldsymbol{E}_{\infty}^{\prime}\right), \boldsymbol{E}_{\infty}^{\prime}=\hat{A}_{\infty} \boldsymbol{E}_{\infty} \\
\hat{\mathbf{A}}_{\infty}=\left(A_{1 \infty}, A_{2 \infty}, A_{3 \infty}\right), \hat{A}_{i \infty}=\frac{\hat{a}_{i \infty}}{\hat{A}_{\infty} \hat{c}_{s \infty}^{2}}, \hat{a}_{i \infty}=\hat{V}_{\infty} \hat{E}_{i \infty}, i=1,2,3
\end{gathered}
$$

Using relations (6.4) and (5.8), we obtain the basic problem(the primes are dropped): one seeks solutions of the systems

$$
\begin{aligned}
& L_{\infty} w_{\infty}+v_{3 \infty}=0, \\
& L_{\infty} s_{\infty}=\rho_{e \infty}, \\
& L_{\infty} v_{i \infty}+\frac{\partial \tilde{p}_{\infty}}{\partial x_{i}}=\hat{A}_{i \infty} \rho_{e \infty}, i=1,2\left(\hat{A}_{2 \infty}=0\right), \\
& L_{\infty} v_{3 \infty}+\hat{\beta}_{\infty} \frac{\partial \tilde{p}_{\infty}}{\partial x_{3}}=\hat{N}_{\infty} \triangle^{2} w_{\infty}-\hat{B}_{\infty} \hat{\lambda}_{\infty}^{2} \frac{\partial^{2} w_{\infty}}{\partial x_{3}^{2}} \\
& -\hat{\sigma}_{\infty} \triangle_{\perp} w_{\infty}+\hat{\Sigma}_{\infty} \frac{\partial s_{\infty}}{\partial x_{3}}, \\
& L_{\infty} \tilde{p}_{\infty}+\operatorname{div}_{\perp} \boldsymbol{v}_{\infty}+\hat{\beta}_{\infty} \frac{\partial v_{3 \infty}}{\partial x_{3}}=R_{1 \infty} \rho_{e \infty}, \\
& \operatorname{curl} \boldsymbol{E}_{\infty}=0 \text {, } \\
& \operatorname{div}\left(\boldsymbol{E}_{\infty} \tilde{\Psi}_{\infty}\right)=4 \pi \rho_{e \infty}, \\
& L_{\infty} \rho_{e \infty}+\left(\hat{\boldsymbol{\Lambda}}_{\infty}, \nabla\right) \rho_{e \infty}=0, \quad t>0, \boldsymbol{x} \in \mathbb{R}_{-}^{3}, \\
& L w+v_{3}=0, \\
& L s=\rho_{e}, \\
& L v_{i}+\frac{\partial \tilde{p}}{\partial x_{i}}=\hat{A}_{i} \rho_{e}, i=1,2\left(\hat{A}_{2}=0\right), \\
& L v_{3}+\hat{\beta} \frac{\partial \tilde{p}}{\partial x_{3}}=\hat{N} \triangle^{2} w-\hat{B} \hat{\lambda}^{2} \frac{\partial^{2} w}{\partial x_{3}^{2}}-\hat{\sigma} \triangle_{\perp} w+\hat{\Sigma} \frac{\partial s}{\partial x_{3}}, \\
& L \tilde{p}+\operatorname{div}_{\perp} \boldsymbol{v}+\hat{\beta} \frac{\partial v_{3}}{\partial x_{3}}=R_{1} \rho_{e}, \\
& \operatorname{curl} \boldsymbol{E}=0 \text {, } \\
& \operatorname{div}(\boldsymbol{E} \tilde{\Psi})=4 \pi \rho_{e}, \\
& L \rho_{e}+(\hat{\boldsymbol{\Lambda}}, \nabla) \rho_{e}=0, \quad t>0, \boldsymbol{x} \in \mathbb{R}_{+}^{3}
\end{aligned}
$$

satisfying the boundary conditions

$$
\begin{gathered}
f_{t}+M_{2} f_{x_{2}}=\frac{M_{1}}{1-\bar{v}_{1}}\left[\frac{v_{1}}{M_{1}}+\tilde{p}+\hat{B} \hat{\gamma} b_{3}-R_{1} s\right], \\
{[w]=0,}
\end{gathered}
$$




$$
\begin{aligned}
& v_{1}+\frac{1+M_{1}^{2}}{2 M_{1}} \tilde{p}-\frac{M_{1} R_{1}}{2} s+\frac{M_{1} \hat{B} \hat{\gamma} b_{3}}{2}+\frac{\hat{W}}{2}\left(1-2 \tilde{\Psi}_{11}\right) E_{1} \\
& -\frac{\hat{W} \sum_{i=2}^{3} \tilde{\Psi}_{i 1} E_{i}}{2}+\frac{W \hat{W} \sum_{i=2}^{3} \tilde{\Psi}_{1 i} f_{x_{i}}}{2}=\frac{1}{K_{\infty}}\left(v_{1 \infty}+\frac{1+M_{1 \infty}^{2}}{2 M_{1 \infty}} \tilde{p}_{\infty}\right. \\
& -\frac{M_{1 \infty} R_{1 \infty}}{2} s_{\infty}+\frac{M_{1 \infty} \hat{B}_{\infty} \hat{\gamma}_{\infty} b_{3 \infty}}{2}+\frac{\hat{W}_{\infty}}{2}\left(1-2 \tilde{\Psi}_{11 \infty}\right) E_{1 \infty} \\
& \left.-\frac{\hat{W}_{\infty} \sum_{i=2}^{3} \tilde{\Psi}_{i 1 \infty} E_{i \infty}}{2}+\frac{W_{\infty} \hat{W}_{\infty} \sum_{i=2}^{3} \tilde{\Psi}_{1 i \infty} f_{x_{i}}}{2}\right), \\
& v_{2}+\hat{W} \tilde{\Psi}_{11} E_{2}-\frac{1}{K_{\infty}}\left(v_{2 \infty}+\hat{W}_{\infty} \tilde{\Psi}_{11 \infty} E_{2 \infty}\right) \\
& =\left(\hat{\mu}-W \hat{W} \tilde{\Psi}_{11}+\frac{W_{\infty} \hat{W}_{\infty} \tilde{\Psi}_{11 \infty}}{K_{\infty}}\right) f_{x_{2}}, \\
& v_{3}+\hat{W} \tilde{\Psi}_{11} E_{3}-\frac{\hat{N}}{M_{1}} \triangle b_{1}+\frac{\hat{\sigma}}{M_{1}} b_{1}-\frac{1}{K_{\infty}}\left(v_{3 \infty}+\hat{W}_{\infty} \tilde{\Psi}_{11 \infty} E_{3 \infty}\right. \\
& \left.-\frac{\hat{N}_{\infty}}{M_{1 \infty}} \triangle b_{1 \infty}+\frac{\hat{\sigma}_{\infty}}{M_{1 \infty}} b_{1 \infty}\right)=\left(\hat{\mu}-W \hat{W} \tilde{\Psi}_{11}+\frac{W_{\infty} \hat{W}_{\infty} \tilde{\Psi}_{11 \infty}}{K_{\infty}}\right) f_{x_{3}}, \\
& \left(1+\frac{M_{1}^{2}}{\bar{v}_{1}}\right) \tilde{p}+\left(M_{1}\left(1+\frac{1}{\bar{v}_{1}}\right)\right) v_{1}+\left(D-R_{1} \frac{M_{1}^{2}}{\bar{v}_{1}}\right) s+\frac{M_{1}^{2}}{\bar{v}_{1}} \hat{B} \hat{\gamma} b_{3} \\
& -\frac{1}{K_{\infty}^{2}}\left(\left(1+M_{1 \infty} M_{1} K_{\infty}\right) \tilde{p}_{\infty}+\left(M_{1 \infty}+M_{1} K_{\infty}\right) v_{1 \infty}\right. \\
& \left.+\left(D_{\infty}-R_{1 \infty} M_{1} M_{1 \infty} K_{\infty}\right) s_{\infty}\right)-\frac{M_{1}^{2}}{\bar{v}_{1}} \hat{B}_{\infty} \hat{\gamma}_{\infty} b_{3 \infty}=0, \\
& -\left(M_{1}+\hat{\Lambda}_{1}\right) \rho_{e}+\frac{\bar{A}}{K_{\infty}}\left(M_{1 \infty}+\hat{\Lambda}_{1 \infty}\right) \rho_{e \infty}=\hat{\chi} \frac{\partial \omega}{\partial t}, \\
& -\sum_{i=1}^{3} E_{i} \tilde{\Psi}_{i 1}+W\left(\tilde{\Psi}_{12} f_{x_{2}}+\tilde{\Psi}_{13} f_{x_{3}}\right)+4 \pi \hat{\chi} \omega \\
& =\bar{A}\left(-\sum_{i=1}^{3} E_{i \infty} \tilde{\Psi}_{i 1 \infty}+W_{\infty}\left(\tilde{\Psi}_{12 \infty} f_{x_{2}}+\tilde{\Psi}_{13 \infty} f_{x_{3}}\right)\right), \\
& W f_{x_{k}}+E_{k}=\bar{A}\left(W_{\infty} f_{x_{k}}+E_{k \infty}\right), k=2,3 .
\end{aligned}
$$

for $t>0, x_{1}=0, \boldsymbol{x}=\left(x_{2}, x_{3}\right) \in \mathbb{R}^{2}$. Here $f=f\left(t, x_{2}, x_{3}\right)$ is a small disturbance of the shock front,

$$
\begin{gathered}
L=\frac{\partial}{\partial t}+M_{1} \frac{\partial}{\partial x_{1}}+M_{2} \frac{\partial}{\partial x_{2}}, M_{i}=\frac{\hat{v}_{i}}{\hat{c}_{s}}, i=1,2, \\
L_{\infty}=K_{\infty} \frac{\partial}{\partial t}+M_{1 \infty} \frac{\partial}{\partial x_{1}}+M_{2 \infty} \frac{\partial}{\partial x_{2}}, M_{i \infty}=\frac{\hat{v}_{i \infty}}{\hat{c}_{s \infty}}, i=1,2, \\
\hat{\boldsymbol{\xi}}_{\infty}=\hat{\boldsymbol{E}}_{\infty} \hat{\Omega}_{\infty}, \\
\mathbb{R}_{-}^{3}=\left\{\boldsymbol{x} \in \mathbb{R}^{3}, x_{1}<0\right\}, \quad \mathbb{R}_{+}^{3}=\left\{\boldsymbol{x} \in \mathbb{R}^{3}, x_{1}>0\right\},
\end{gathered}
$$




$$
\begin{gathered}
\bar{v}_{1}=\frac{\hat{v}_{1}}{\hat{v}_{1 \infty}}, \hat{\mu}=M_{1} \frac{1-\bar{v}_{1}}{\bar{v}_{1}}, D=\frac{\hat{T} \hat{s}}{\hat{c}_{s}^{2}}, \hat{T}=\left(\hat{\tilde{U}}_{0}\right)_{s}, D_{\infty}=\frac{\hat{T}_{\infty} \hat{s}_{\infty}}{\hat{c}_{s \infty}^{2}}, \hat{T}_{\infty}=\left(\hat{\tilde{U}}_{0 \infty}\right)_{s \infty}, \\
K_{\infty}=\frac{\hat{c}_{s}}{\hat{c}_{s \infty}}, M_{1 \infty}=\frac{\hat{v}_{1 \infty}}{\hat{c}_{s \infty}}, M_{2 \infty}=\frac{\hat{v}_{2 \infty}}{\hat{c}_{s \infty}} \\
W=\hat{E}_{1} \hat{A}, W_{\infty}=\hat{E}_{1 \infty} \hat{A}_{\infty}, \hat{W}=\frac{W}{4 \pi \hat{\rho}_{1} \hat{c}_{s} \hat{A}^{2}}, \hat{W}_{\infty}=\frac{W_{\infty}}{4 \pi \hat{\rho}_{\infty} \hat{v}_{1 \infty} \hat{c}_{s \infty} \hat{A}_{\infty}^{2}} \\
\bar{A}=\frac{\hat{A}}{\hat{A}_{\infty}}, \hat{\chi}=\hat{\omega} \hat{A}
\end{gathered}
$$

REMARK 6.1. Let the quantities $\hat{r}, \hat{r}_{\infty}, \tilde{\Psi}_{11}, \tilde{\Psi}_{11 \infty}$ be such that

$$
\hat{r}=\alpha \tilde{\Psi}_{11}^{2}, \hat{r}_{\infty}=\alpha \tilde{\Psi}_{11 \infty}^{2}
$$

i.e.,

$$
\begin{gathered}
\tilde{\Psi}_{11}=\frac{(2 \pi-1) \pm \sqrt{(2 \pi-1)^{2}+8 \alpha \pi}}{8 \alpha \pi} \\
\tilde{\Psi}_{11 \infty}=\frac{(2 \pi-1) \pm \sqrt{(2 \pi-1)^{2}+8 \alpha \pi}}{8 \alpha \pi}
\end{gathered}
$$

where $\alpha$ is a constant. Then

$$
\hat{\triangle}=\left[\hat{r} \hat{E}_{1}^{2}\right]=\alpha\left[\tilde{\Psi}_{11}^{2} \hat{E}_{1}^{2}\right]=\alpha\left(\tilde{\Psi}_{11} \hat{E}_{1}+\tilde{\Psi}_{11 \infty} \hat{E}_{1 \infty}\right)\left[\tilde{\Psi}_{11} \hat{E}_{1}\right]=\alpha\left(2 \tilde{\Psi}_{11} \hat{E}_{1}-4 \pi \hat{\omega}\right) 4 \pi \hat{\omega}
$$

and $\hat{\triangle}$ is small if $\hat{\omega}$ is small or $\hat{\omega}$ is close to $\frac{\tilde{\Psi}_{11} \hat{E}_{1}}{2 \pi}$.

Let

$$
\tilde{U}_{0}=\tilde{U}_{0}(V, s)
$$

obey the state equation for a polytropic gas [12, 13]:

$$
\tilde{U}_{0}(V, s)=\tilde{C} \frac{\exp \left(\frac{s}{\tilde{c}_{V}}\right)}{(\tilde{\gamma}-1) V^{\tilde{\gamma}-1}},
$$

where

$\tilde{C}>0$ is a constant,

$\tilde{c}_{V}>0$ is the unit heat capacity of the gas,

$\tilde{\gamma}>1$ is the adiabatic index.

If $|\hat{\triangle}| \ll 1$, then the analysis of relations (6.4) (see also [3, 14]) leads to the following inequalities describing the piecewise constant solution (6.2):

$$
\begin{gathered}
\hat{v}_{1 \infty}>\hat{c}_{\infty}, \quad 0<\hat{v}_{1}<\hat{c}, \\
\hat{\tilde{p}}>\hat{\tilde{p}}_{\infty}>0, \quad \hat{\rho}>\hat{\rho}_{\infty}>0, \\
\hat{v}_{1 \infty}>\hat{v}_{1}, \quad \hat{s}>\hat{s}_{\infty} .
\end{gathered}
$$

Here

$$
\hat{c}^{2}=\tilde{\gamma} \hat{\tilde{p}} \hat{V}, \quad \hat{c}_{\infty}^{2}=\tilde{\gamma} \hat{\tilde{p}}_{\infty} \hat{V}_{\infty} .
$$

Thus, from the physical point of view the piecewise constant solution (6.2) of the equations of a layered dielectric described above means that we have a shock wave that 
separates a supersonic stationary incident flow (the Mach number ahead of the shock wave

$$
\hat{M}_{1 \infty}=\frac{\hat{v}_{1 \infty}}{\hat{c}_{\infty}}>1, \quad \text { see }(\underline{6.8})
$$

from a subsonic stationary flow behind the shock wave (the Mach number behind the shock wave

$$
\hat{M}_{1}=\frac{\hat{v}_{1}}{\hat{c}}<1, \quad \text { see }(\underline{6.8}) \text {. }
$$

Remark 6.2. Problem 6.5a -6.5h), 6.6a -6.6h), 6.7a -6.7i can be somewhat simplified if we make the Galilean transform

$$
t^{\prime}=t, \quad x_{2}^{\prime}=x_{2}-M_{2} t .
$$

As a result, the operators $L, L_{\infty}$ in the systems 6.5a 6.5h, 6.6a 6.6h take the form (the primes are dropped)

$$
\begin{gathered}
L=\frac{\partial}{\partial t}+M_{1} \frac{\partial}{\partial x_{1}} \\
L_{\infty}=K_{\infty} \frac{\partial}{\partial t}+M_{1 \infty} \frac{\partial}{\partial x_{1}}
\end{gathered}
$$

and the term $\left(f_{t}+M_{2} f_{x_{2}}\right)$ appearing in the boundary condition (6.7a) becomes $f_{t}$.

REMARK 6.3. The basic problem formulated above is written so that it permits jumps in the constant components $B, N, \omega_{\perp}, \omega_{\|}$of the matrix $\tilde{\Psi}$.

REMARK 6.4. Further we will assume that

$$
M_{1 \infty}+\hat{\Lambda}_{1 \infty} \geqslant 0 .
$$

If condition (6.9) is satisfied for equation (6.5h) we do not need to set boundary conditions at $x_{1}=0$ (the function $\rho_{e \infty}(t, \boldsymbol{x}), x_{1}<0$ is completely defined by the initial data $\left.\left.\rho_{e \infty}\right|_{t=0}, x_{1}<0\right)$. Therefore, without loss of generality, we assume that

$$
\rho_{e \infty}(t, \boldsymbol{x}) \equiv 0, \quad x_{1}<0 .
$$

Similar arguments are correct for equation (6.5b) as well, i.e., without loss of generality, we also assume that

$$
s_{\infty}(t, \boldsymbol{x}) \equiv 0, \quad x_{1}<0 .
$$

Therefore, taking into account (6.10), 6.11), the basic problem can be rewritten as follows. We seek solutions of the system

$$
\begin{gathered}
L_{\infty} w_{\infty}+v_{3 \infty}=0 \\
L_{\infty} v_{i \infty}+\frac{\partial \tilde{p}_{\infty}}{\partial x_{i}}=0, i=1,2 \\
L_{\infty} v_{3 \infty}+\hat{\beta}_{\infty} \frac{\partial \tilde{p}_{\infty}}{\partial x_{3}}=\hat{N}_{\infty} \triangle^{2} w_{\infty}-\hat{B}_{\infty} \hat{\lambda}_{\infty}^{2} \frac{\partial^{2} w_{\infty}}{\partial x_{3}^{2}}-\hat{\sigma}_{\infty} \triangle_{\perp} w_{\infty} \\
L_{\infty} \tilde{p}_{\infty}+\operatorname{div}_{\perp} \boldsymbol{v}_{\infty}+\hat{\beta}_{\infty} \frac{\partial v_{3 \infty}}{\partial x_{3}}=0 \\
\operatorname{curl} \boldsymbol{E}_{\infty}=0 \\
\operatorname{div}\left(\boldsymbol{E}_{\infty} \tilde{\Psi}_{\infty}\right)=0, \quad t>0, \boldsymbol{x} \in \mathbb{R}_{-}^{3} \\
L w+v_{3}=0
\end{gathered}
$$




$$
\begin{gathered}
L s=\rho_{e}, \\
L v_{i}+\frac{\partial \tilde{p}}{\partial x_{i}}=\hat{A}_{i} \rho_{e}, i=1,2 \quad\left(\hat{A}_{2}=0\right), \\
L v_{3}+\hat{\beta} \frac{\partial \tilde{p}}{\partial x_{3}}=\hat{N} \triangle^{2} w-\hat{B} \hat{\lambda}^{2} \frac{\partial^{2} w}{\partial x_{3}^{2}}-\hat{\sigma} \triangle_{\perp} w+\hat{\Sigma} \frac{\partial s}{\partial x_{3}}, \\
L \tilde{p}+\operatorname{div}_{\perp} \boldsymbol{v}+\hat{\beta} \frac{\partial v_{3}}{\partial x_{3}}=R_{1} \rho_{e}, \\
\operatorname{curl} \boldsymbol{E}=0, \\
\operatorname{div}(\boldsymbol{E} \tilde{\Psi})=4 \pi \rho_{e}, \\
L \rho_{e}+(\hat{\boldsymbol{\Lambda}}, \nabla) \rho_{e}=0, \quad t>0, \boldsymbol{x} \in \mathbb{R}_{+}^{3}
\end{gathered}
$$

satisfying the boundary conditions

$$
\begin{aligned}
& f_{t}=\frac{M_{1}}{1-\bar{v}_{1}}\left(\left[\frac{v_{1}}{M_{1}}+\tilde{p}+\hat{B} \hat{\gamma} b_{3}\right]-R_{1} s\right), \\
& {[w]=0,} \\
& v_{1}+\frac{1+M_{1}^{2}}{2 M_{1}} \tilde{p}-\frac{M_{1} R_{1}}{2} s+\frac{M_{1} \hat{B} \hat{\gamma} b_{3}}{2}+\frac{\hat{W}}{2}\left(1-2 \tilde{\Psi}_{11}\right) E_{1} \\
& -\frac{\hat{W} \sum_{i=2}^{3} \tilde{\Psi}_{i 1} E_{i}}{2}+\frac{W \hat{W} \sum_{i=2}^{3} \tilde{\Psi}_{1 i} f_{x_{i}}}{2}=\frac{1}{K_{\infty}}\left(v_{1 \infty}+\frac{1+M_{1 \infty}^{2}}{2 M_{1 \infty}} \tilde{p}_{\infty}\right. \\
& +\frac{M_{1 \infty} \hat{B}_{\infty} \hat{\gamma}_{\infty} b_{3 \infty}}{2}+\frac{\hat{W}_{\infty}}{2}\left(1-2 \tilde{\Psi}_{11 \infty}\right) E_{1 \infty} \\
& \left.-\frac{\hat{W}_{\infty} \sum_{i=2}^{3} \tilde{\Psi}_{i 1 \infty} E_{i \infty}}{2}+\frac{W_{\infty} \hat{W}_{\infty} \sum_{i=2}^{3} \tilde{\Psi}_{1 i \infty} f_{x_{i}}}{2}\right), \\
& v_{2}+\hat{W} \tilde{\Psi}_{11} E_{2}-\frac{1}{K_{\infty}}\left(v_{2 \infty}+\hat{W}_{\infty} \tilde{\Psi}_{11 \infty} E_{2 \infty}\right) \\
& =\left(\hat{\mu}-W \hat{W} \tilde{\Psi}_{11}+\frac{W_{\infty} \hat{W}_{\infty} \tilde{\Psi}_{11 \infty}}{K_{\infty}}\right) f_{x_{2}}, \\
& v_{3}+\hat{W} \tilde{\Psi}_{11} E_{3}-\frac{\hat{N}}{M_{1}} \triangle b_{1}+\frac{\hat{\sigma}}{M_{1}} b_{1}-\frac{1}{K_{\infty}}\left(v_{3 \infty}+\hat{W}_{\infty} \tilde{\Psi}_{11 \infty} E_{3 \infty}\right. \\
& \left.-\frac{\hat{N}_{\infty}}{M_{1 \infty}} \triangle b_{1 \infty}+\frac{\hat{\sigma}_{\infty}}{M_{1 \infty}} b_{1 \infty}\right)=\left(\hat{\mu}-W \hat{W} \tilde{\Psi}_{11}+\frac{W_{\infty} \hat{W}_{\infty} \tilde{\Psi}_{11 \infty}}{K_{\infty}}\right) f_{x_{3}} \\
& \left(1+\frac{M_{1}^{2}}{\bar{v}_{1}}\right) \tilde{p}+\left(M_{1}\left(1+\frac{1}{\bar{v}_{1}}\right)\right) v_{1}+\left(D-R_{1} \frac{M_{1}^{2}}{\bar{v}_{1}}\right) s+\frac{M_{1}^{2}}{\bar{v}_{1}} \hat{B} \hat{\gamma} b_{3} \\
& -\frac{1}{K_{\infty}^{2}}\left(\left(1+M_{1 \infty} M_{1} K_{\infty}\right) \tilde{p}_{\infty}+\left(M_{1 \infty}+M_{1} K_{\infty}\right) v_{1 \infty}\right) \\
& -\frac{M_{1}^{2}}{\bar{v}_{1}} \hat{B}_{\infty} \hat{\gamma}_{\infty} b_{3 \infty}=0, \\
& \frac{\partial \omega}{\partial t}=-\frac{\left(M_{1}+\hat{\Lambda}_{1}\right)}{\hat{\chi}} \rho_{e},
\end{aligned}
$$




$$
\begin{gathered}
-\sum_{i=1}^{3} E_{i} \tilde{\Psi}_{i 1}+W\left(\tilde{\Psi}_{12} f_{x_{2}}+\tilde{\Psi}_{13} f_{x_{3}}\right)+4 \pi \hat{\chi} \omega \\
=\bar{A}\left(-\sum_{i=1}^{3} E_{i \infty} \tilde{\Psi}_{i 1 \infty}+W_{\infty}\left(\tilde{\Psi}_{12 \infty} f_{x_{2}}+\tilde{\Psi}_{13 \infty} f_{x_{3}}\right)\right), \\
W f_{x_{k}}+E_{k}=\bar{A}\left(W_{\infty} f_{x_{k}}+E_{k \infty}\right), k=2,3 .
\end{gathered}
$$

for $t>0, x_{1}=0, \boldsymbol{x}^{\prime} \in \mathbb{R}^{2}$.

REMARK 6.5. If $\hat{N}_{\infty}=0$, then the basic problem (6.12a)-6.12f , 6.6a)-6.6h), (6.13a) -6.13f) formulated in Remark 5.4 can be somewhat simplified. Indeed, system (6.12a) - (6.12d) can be rewritten as

$$
\mathrm{A}_{\infty}\left(\boldsymbol{U}_{\infty}\right)_{t}+\sum_{k=1}^{3} \mathrm{~B}_{k \infty}\left(\boldsymbol{U}_{\infty}\right)_{x_{k}}+\boldsymbol{\Gamma}_{\infty}=0 .
$$

Here

$$
\boldsymbol{U}_{\infty}=\left(\begin{array}{c}
\boldsymbol{v}_{\infty} \\
\tilde{p}_{\infty} \\
w_{\infty} \\
\boldsymbol{b}_{\infty}
\end{array}\right), \quad \mathrm{B}_{k \infty}=\left(\begin{array}{cc}
\mathrm{R}_{k \infty} & O_{4} \\
O_{4} & \mathrm{~F}_{k \infty}
\end{array}\right), \quad k=1,2,3,
$$

$\mathrm{A}_{\infty}=K_{\infty} \operatorname{diag}\left(1,1,1,1,1, \hat{\sigma}_{\infty}, \hat{\sigma}_{\infty}, \hat{B}_{\infty} \hat{\lambda}_{\infty}^{2}\right)$ is the diagonal matrix,

$$
\begin{gathered}
\mathrm{R}_{1 \infty}=\left(\begin{array}{cccc}
M_{1 \infty} & 0 & 0 & 1 \\
0 & M_{1 \infty} & 0 & 0 \\
0 & 0 & M_{1 \infty} & 0 \\
1 & 0 & 0 & M_{1 \infty}
\end{array}\right), \quad \mathrm{R}_{2 \infty}=\left(\begin{array}{cccc}
0 & 0 & 0 & 0 \\
0 & 0 & 0 & 1 \\
0 & 0 & 0 & 0 \\
0 & 1 & 0 & 0
\end{array}\right), \\
\mathrm{R}_{3 \infty}=\left(\begin{array}{cccc}
0 & 0 & 0 & 0 \\
0 & 0 & 0 & 0 \\
0 & 0 & 0 & \hat{\beta}_{\infty} \\
0 & 0 & \hat{\beta}_{\infty} & 0
\end{array}\right),
\end{gathered}
$$

$\mathrm{F}_{1 \infty}=M_{1 \infty} \operatorname{diag}\left(1, \hat{\sigma}_{\infty}, \hat{\sigma}_{\infty}, \hat{B}_{\infty} \hat{\lambda}_{\infty}^{2}\right)$, the diagonal matrix,

$\mathrm{F}_{2 \infty}, \mathrm{F}_{3 \infty}=O_{4}, O_{4}$ the zero matrix of order 4 ,

$$
\boldsymbol{\Gamma}_{\infty}=\left(0,0, \hat{B}_{\infty} \hat{\lambda}_{\infty}^{2} \frac{\partial^{2} w_{\infty}}{\partial x_{3}^{2}}+\hat{\sigma}_{\infty} \triangle_{\perp} w_{\infty}, 0, v_{3 \infty}, \hat{\sigma}_{\infty} \frac{\partial v_{3 \infty}}{\partial x_{1}}, \hat{\sigma}_{\infty} \frac{\partial v_{3 \infty}}{\partial x_{2}}, \hat{B}_{\infty} \hat{\lambda}_{\infty}^{2} \frac{\partial v_{3 \infty}}{\partial x_{3}}\right)^{\top} .
$$

We multiply system (6.14) scalar-wise by $2 \boldsymbol{U}_{\infty}$ :

$$
\begin{gathered}
\left(\boldsymbol{U}_{\infty}, \mathrm{A}_{\infty} \boldsymbol{U}_{\infty}\right)_{t}+\sum_{k=1}^{3}\left(\boldsymbol{U}_{\infty}, \mathrm{B}_{k \infty} \boldsymbol{U}_{\infty}\right)_{x_{k}}+2 \hat{B}_{\infty} \hat{\lambda}_{\infty}^{2} \frac{\partial}{\partial x_{3}}\left(v_{3 \infty} b_{3 \infty}\right) \\
+2 \hat{\sigma}_{\infty} \sum_{l=1}^{2} \frac{\partial}{\partial x_{l}}\left(v_{3 \infty} b_{l \infty}\right)+2 v_{3 \infty} w_{\infty}=0 .
\end{gathered}
$$

Then, we integrate (6.15) over the domain $\mathbb{R}_{-}^{3}$ :

$$
\frac{d J(t)}{d t}+\left.\left\{\left(\boldsymbol{U}_{\infty}, \mathrm{B}_{1 \infty} \boldsymbol{U}_{\infty}\right)+2 \hat{\sigma}_{\infty} v_{3 \infty} b_{1 \infty}\right\}\right|_{x_{1}=0}+2 \int_{\mathbb{R}_{-}^{3}} v_{3_{\infty}} w_{\infty} d \boldsymbol{x}=0
$$


where

$$
\begin{gathered}
\left(\boldsymbol{U}_{\infty}, \mathrm{B}_{1 \infty} \boldsymbol{U}_{\infty}\right)=M_{1 \infty}\left(v_{1 \infty}^{2}+v_{2 \infty}^{2}+v_{3 \infty}^{2}+\tilde{p}_{\infty}^{2}+w_{\infty}^{2}+\hat{\sigma}_{\infty} b_{1 \infty}^{2}+\hat{\sigma}_{\infty} b_{2 \infty}^{2}\right. \\
\left.+\hat{B}_{\infty} \hat{\lambda}_{\infty}^{2} b_{3 \infty}^{2}\right)+2 v_{1 \infty} \tilde{p}_{\infty}, \\
J(t)=\int_{\mathbb{R}_{-}^{3}}\left(\boldsymbol{U}_{\infty}, \mathrm{A}_{\infty} \boldsymbol{U}_{\infty}\right) d \boldsymbol{x} .
\end{gathered}
$$

While getting (6.16) we assumed that $\left|\boldsymbol{U}_{\infty}\right| \rightarrow 0$ as $|\boldsymbol{x}| \rightarrow \infty$. Let

$$
\left.\begin{array}{l}
M_{1 \infty}=\hat{M}_{1 \infty} \hat{\lambda}_{\infty} \geq 1, \\
M_{1 \infty}^{2} \geq \hat{\sigma}_{\infty} .
\end{array}\right\}
$$

Then

and (6.16) implies

$$
\left.\left\{\left(\boldsymbol{U}_{\infty}, \mathrm{B}_{1 \infty} \boldsymbol{U}_{\infty}\right)+2 \hat{\sigma}_{\infty} v_{3 \infty} b_{1 \infty}\right\}\right|_{x_{1}=0} \geq 0
$$

i.e.,

$$
\frac{d J(t)}{d t} \leq K_{0} J(t)
$$

$$
J(t) \leq e^{K_{0} t} J(0) .
$$

Here $K_{0}>0$ is a constant. It obviously follows from (6.17) that if $\left.\boldsymbol{U}_{\infty}\right|_{t=0}=0$, then

$$
\boldsymbol{U}_{\infty}(t, \boldsymbol{x})=0 \quad\left(x_{1}<0\right)
$$

for all $t>0$. Taking this into account, we can rewrite the basic problem (we assume that the matrix $\tilde{\Psi}$ is diagonal).

Version of the basic problem. We seek solutions of system (6.12e $-(6.12 \mathrm{f})$ for $t>0, \quad x \in \mathbb{R}_{-}^{3}$, of system (6.6a)- $6.6 \mathrm{~h}$ ) for $t>0, \boldsymbol{x} \in \mathbb{R}_{+}^{3}$, satisfying the following boundary conditions for $t>0, x_{1}=0, \boldsymbol{x}^{\prime} \in \mathbb{R}^{2}$ :

$$
\begin{gathered}
w=0, \\
f_{t}=\frac{\mu}{\hat{\mu}} \tilde{p}-\frac{\mu_{E}}{\hat{\mu}} E_{1}+\frac{\mu_{E \infty}}{\hat{\mu}} E_{1 \infty}, \\
v_{1}+d M_{1} \tilde{p}+d_{E} M_{1} E_{1}-d_{E \infty} M_{1} E_{1 \infty}=0, \\
v_{2}+\Theta E_{2}=\bar{\mu} f_{x_{2}}, \\
\left(M_{1}^{2}-\hat{\sigma}\right) b_{1}+\hat{N} \triangle b_{1}-M_{1} \Theta E_{3}+M_{1} \bar{\mu} f_{x_{3}}=0, \\
s=\nu \tilde{p}+\nu_{E} E_{1}-\nu_{E \infty} E_{1 \infty}, \\
\frac{\partial \omega}{\partial t}=-\frac{M_{1}+\hat{\Lambda}_{1}}{\hat{\chi}} \rho_{e}, \\
\omega=\frac{\tilde{\Psi}}{4 \pi \hat{\chi}} E_{1}-\frac{\bar{A} \tilde{\Psi}_{11 \infty}}{4 \pi \hat{\chi}} E_{1 \infty}, \\
E_{k \infty}=\frac{E_{k}}{\bar{A}}+\left(\frac{W}{\bar{A}}-W_{\infty}\right) f_{x_{k}}, k=2,3,
\end{gathered}
$$

where

$$
\nu=\frac{\beta^{2} \hat{\mu}}{2 M_{1}\left(D-\frac{M_{1} R_{1} \hat{\mu}}{2}\right)}, \quad \beta^{2}=1-M_{1}^{2},
$$




$$
\begin{gathered}
\nu_{E}=\frac{\left(M_{1}+\frac{M_{1}}{\bar{v}_{1}}\right)\left(1-2 \tilde{\Psi}_{11}\right) \hat{W}}{2\left(D-\frac{M_{1} R_{1} \hat{\mu}}{2}\right)}, \quad \nu_{E \infty}=\frac{\left(M_{1}+\frac{M_{1}}{\bar{v}_{1}}\right)\left(1-2 \tilde{\Psi}_{11 \infty}\right) \hat{W}_{\infty}}{2 K_{\infty}\left(D-\frac{M_{1} R_{1} \hat{\mu}}{2}\right)}, \\
M_{1} d=\frac{1+M_{1}^{2}}{2 M_{1}}-\frac{M_{1} R_{1}}{2} \nu \\
M_{1} d_{E}=\frac{\hat{W}}{2}\left(1-2 \tilde{\Psi}_{11}\right)-\frac{M_{1} R_{1}}{2} \nu_{E}, \quad M_{1} d_{E \infty}=\frac{\hat{W}_{\infty}}{2 K_{\infty}}\left(1-2 \tilde{\Psi}_{11 \infty}\right)-\frac{M_{1} R_{1}}{2} \nu_{E \infty}, \\
\mu=M_{1}^{2} \frac{1-d-R_{1} \nu}{\bar{v}_{1}}, \quad \mu_{E}=M_{1}^{2} \frac{d_{E}+R_{1} \nu_{E}}{\bar{v}_{1}}, \quad \mu_{E \infty} \frac{d_{E \infty}+R_{1} \nu_{E \infty}}{\bar{v}_{1}} \\
\bar{\mu}=\hat{\mu}-W \hat{W} \tilde{\Psi}_{11}+\frac{W \hat{W}_{\infty} \tilde{\Psi}_{11 \infty}}{\bar{A} K_{\infty}}, \quad \Theta=\hat{W} \tilde{\Psi}_{11}-\frac{\hat{W}_{\infty} \tilde{\Psi}_{11 \infty}}{\bar{A} K_{\infty}} .
\end{gathered}
$$

7. Ill-posedness of the stability problem for shock waves. Following [10, 11, 14, the question on the stability of shock waves for the electrohydrodynamical model of a layered dielectric is reduced to the study of the well-posedness of the linearized stability problem (see the basic problem (6.5a)-6.7f) from Section 5). The proof of illposedness follows from the construction of Hadamard-type ill-posedness examples (see [15, 16]). From the physical point of view the presence of such examples means the instability of shock waves in layered structures.

As usual (see [17]), in the case of linear problems we construct Hadamard examples by seeking particular exponential solutions. In this work we restrict ourselves to the case of the version of the basic problem (the general case is postponed to the future). For the 2D case the version of the basic problem looks as follows (see Section $5)$. One seeks solutions of the system

$$
\begin{gathered}
\left(E_{1 \infty}\right)_{z}-\left(E_{3 \infty}\right)_{x}=0 \\
\left(E_{1 \infty} \tilde{\Psi}_{11 \infty}\right)_{x}+\left(E_{3 \infty} \tilde{\Psi}_{33 \infty}\right)_{z}=0, t>0,(x, z) \in \mathbb{R}_{-}^{2} ; \\
L w+v_{3}=0 \\
L s=\rho_{e} \\
L v_{1}+\frac{\partial \tilde{p}}{\partial x}=\hat{A}_{1} \rho_{e} \\
L v_{3}+\hat{\beta} \frac{\partial \tilde{p}}{\partial z}=\hat{N} \triangle^{2} w-\hat{B} \hat{\lambda}^{2} \frac{\partial^{2} w}{\partial z^{2}}-\hat{\sigma} \frac{\partial^{2} w}{\partial x^{2}}+\hat{\Sigma} \frac{\partial s}{\partial z}, \\
L \tilde{p}+\frac{\partial v_{1}}{\partial x}+\hat{\beta} \frac{\partial v_{3}}{\partial z}=R_{1} \rho_{e} \\
\left(E_{1}\right)_{z}-\left(E_{3}\right)_{x}=0 \\
\left(E_{1} \tilde{\Psi}_{11}\right)_{x}+\left(E_{3} \tilde{\Psi}_{33}\right)_{z}=4 \pi \rho_{e} \\
L \rho_{e}+\hat{\Lambda}_{1} \frac{\partial \rho_{e}}{\partial x}=0, t>0,(x, z) \in \mathbb{R}_{+}^{2}
\end{gathered}
$$


satisfying the boundary conditions

$$
\begin{gathered}
w=0, \\
f_{t}=\frac{\mu}{\hat{\mu}} \tilde{p}-\frac{\mu_{E}}{\hat{\mu}} E_{1}+\frac{\mu_{E \infty}}{\hat{\mu}} E_{1 \infty}=0, \\
v_{1}+d M_{1} \tilde{p}+d_{E} M_{1} E_{1}-d_{E \infty} M_{1} E_{1 \infty}=0, \\
\left(M_{1}^{2}-\hat{\sigma}\right) b_{1}+\hat{N} \triangle b_{1}-M_{1} \Theta E_{3}+M_{1} \bar{\mu} f_{z}=0, \\
s=\nu \tilde{p}+\nu_{E} E_{1}-\nu_{E \infty} E_{1 \infty}, \\
\omega_{t}=-\nu_{\omega} \rho_{e}, \\
\omega=\frac{1}{4 \pi \hat{\chi}}\left(\tilde{\Psi}_{11} E_{1}-\bar{A} \tilde{\Psi}_{11 \infty} E_{1 \infty}\right), \\
E_{3 \infty}=\frac{E_{3}}{\bar{A}}+W_{E} f_{z}
\end{gathered}
$$

for $t>0, x=0, z \in \mathbb{R}_{1}$. Here

$$
\begin{gathered}
x_{1}=x, x_{3}=z, b_{1}=\frac{\partial w}{\partial x}, \\
\nu_{\omega}=\frac{M_{1}+\hat{\Lambda}_{1}}{\hat{\chi}}, W_{E}=\frac{W}{A}-W_{\infty} .
\end{gathered}
$$

We seek solutions of system (7.1) in the form

$$
\left(\begin{array}{c}
E_{1 \infty} \\
E_{3 \infty}
\end{array}\right)(t, x, z)=\left(\begin{array}{l}
\mathcal{E}_{1 \infty} \\
\mathcal{E}_{3 \infty}
\end{array}\right) \exp \{n(\tau t+r x+i z)\}, x<0
$$

and solutions of system (7.2) in the form

$$
\left(\begin{array}{c}
w \\
s \\
v_{1} \\
v_{3} \\
\tilde{p} \\
E_{1} \\
E_{3} \\
\rho_{e}
\end{array}\right)(t, x, z)=\left(\begin{array}{c}
W_{0} \\
S \\
V_{1} \\
V_{3} \\
\tilde{\mathcal{P}} \\
\mathcal{E}_{1} \\
\mathcal{E}_{3} \\
R_{e}
\end{array}\right) \exp \{n(\tau t+R x+i z)\}, x>0
$$

where $\mathcal{E}_{1 \infty}, \mathcal{E}_{3 \infty}, W_{0}, S, V_{1}, V_{3}, \tilde{\mathcal{P}}, \mathcal{E}_{1}, \mathcal{E}_{3}, R_{e}, \tau, r, R$ are constants, with

$$
\operatorname{Re} \tau>0, \operatorname{Re} r>0, \operatorname{Re} R<0, n \in \mathbb{Z} .
$$

The small perturbations of the shock front and the surface charge are sought in the form

$$
\begin{aligned}
& f(t, z)=F \exp \{n(\tau t+i z)\}, \\
& \omega(t, z)=\Omega \exp \{n(\tau t+i z)\} .
\end{aligned}
$$

Here $F$ and $\Omega$ are constants.

Substituting expression (7.4) into system (7.1), we obtain the following linear algebraic system for $\mathcal{E}_{1 \infty}$ and $\mathcal{E}_{3 \infty}$ :

$$
i \mathcal{E}_{1 \infty}-r \mathcal{E}_{3 \infty}=0
$$




$$
\tilde{\Psi}_{11 \infty} r \mathcal{E}_{1 \infty}+\tilde{\Psi}_{33 \infty i \mathcal{E}_{3 \infty}=0}
$$

i.e.,

$$
\mathrm{A}_{\infty}\left(\begin{array}{l}
\mathcal{E}_{1 \infty} \\
\mathcal{E}_{3 \infty}
\end{array}\right)=0
$$

where

$$
\mathrm{A}_{\infty}=\left(\begin{array}{cc}
i & -r \\
\tilde{\Psi}_{11 \infty} r & \tilde{\Psi}_{33 \infty} i
\end{array}\right)
$$

If $\operatorname{det} \mathrm{A}_{\infty}=0$, i.e.,

$$
r^{2}=\frac{\tilde{\Psi}_{33 \infty}}{\tilde{\Psi}_{11 \infty}}=\tilde{\alpha}_{\infty}
$$

then system (7.8) has a nontrivial solution. Let $\tilde{\alpha}_{\infty}>0$. Considering (7.9) as an equation for determining $r$, we find its root with the desired property (see (7.6)):

$$
r=r_{+}=+\sqrt{\tilde{\alpha}_{\infty}} .
$$

Substituting expression (7.5) into system (7.2), for the quantities $W_{0}, S, V_{1}, V_{3}, \tilde{\mathcal{P}}$, $\mathcal{E}_{1}, \mathcal{E}_{3}$, and $R_{e}$ we obtain the linear algebraic system

$$
\begin{gathered}
\hat{l} \tilde{W}_{0}+V_{3}=0, \hat{l}=\tau+R M_{1}, \tilde{W}_{0}=n W_{0}, \\
\hat{l} S-\varepsilon R_{e}=0, \varepsilon=\frac{1}{n}, \\
\hat{l} V_{1}+R \tilde{\mathcal{P}}-\varepsilon \hat{A}_{1} R_{e}=0, \\
\tilde{q} \tilde{W}_{0}+i \varepsilon^{2} \hat{\Sigma} S-\varepsilon^{2} \hat{l}_{3}-i \varepsilon^{2} \hat{\beta} \tilde{\mathcal{P}}=0, \\
\tilde{q}=\hat{N}\left(R^{2}-1\right)^{2}+\varepsilon^{2}\left(\hat{B} \hat{\lambda}^{2}-\hat{\sigma} R^{2}\right)=\tilde{q}(R), \\
R V_{1}+i \hat{\beta} V_{3}+\hat{l} \tilde{\mathcal{P}}-R_{1} \varepsilon R_{e}=0, \\
i \mathcal{E}_{1}-R \mathcal{E}_{3}=0, \\
\tilde{\Psi}_{11} R \mathcal{E}_{1}+i \tilde{\Psi}_{33} \mathcal{E}_{3}-4 \pi \varepsilon R_{e}=0, \\
\left(\tau+R\left(M_{1}+\hat{\Lambda}_{1}\right)\right) R_{e}=0,
\end{gathered}
$$

i.e.,

$$
\mathrm{A}\left(\begin{array}{c}
\tilde{W}_{0} \\
S \\
V_{1} \\
V_{3} \\
\tilde{\mathcal{P}} \\
\mathcal{E}_{1} \\
\mathcal{E}_{3} \\
R_{e}
\end{array}\right)=0,
$$


where

$$
\mathrm{A}=\left(\begin{array}{cccccccc}
\hat{l} & 0 & 0 & 1 & 0 & 0 & 0 & 0 \\
0 & \hat{l} & 0 & 0 & 0 & 0 & 0 & -\varepsilon \\
0 & 0 & \hat{l} & 0 & R & 0 & 0 & -\varepsilon \hat{A}_{1} \\
\tilde{q} & i \varepsilon^{2} \Sigma & 0 & -\varepsilon^{2} \hat{l} & -i \varepsilon^{2} \hat{\beta} & 0 & 0 & 0 \\
0 & 0 & R & i \hat{\beta} & \hat{l} & 0 & 0 & -\varepsilon R_{1} \\
0 & 0 & 0 & 0 & 0 & i & -R & 0 \\
0 & 0 & 0 & 0 & 0 & \tilde{\Psi}_{11} R & i \tilde{\Psi}_{33} & -4 \pi \varepsilon \\
0 & 0 & 0 & 0 & 0 & 0 & 0 & \hat{l}_{1}
\end{array}\right), \hat{l}_{1}=\tau+R\left(M_{1}+\hat{\Lambda}_{1}\right) .
$$

The system (7.11) has a nontrivial solution if $\operatorname{det} A=0$, i.e.,

$$
\begin{aligned}
& \hat{l}_{1}\left(\tilde{\Psi}_{11} R^{2}-\tilde{\Psi}_{33}\right) \hat{l}\left\{\hat{N}(R+1)^{2}(R-1)^{2}(\hat{l}-R)(\hat{l}+R)\right. \\
& \left.-\varepsilon^{2}\left[\hat{l}^{2}\left(R^{2}-\hat{l}^{2}-\hat{\beta}^{2}\right)-\left(\hat{l}^{2}-R^{2}\right)\left(\hat{B} \hat{\lambda}^{2}-\hat{\sigma}^{2} R^{2}\right)\right]\right\}=0 .
\end{aligned}
$$

We assume that the quantity $\tau$ is known and represented in the form of the series

$$
\tau=\tau_{0}+\varepsilon \tau_{1}+\ldots, \operatorname{Re} \tau_{0}>0 .
$$

Considering (7.12) as an equation for $R$, we find its roots with the desired property (see (7.6) ):

a) if $M_{1}+\hat{\Lambda}_{1}>0$, then

$$
\begin{gathered}
R_{0}=-\frac{\tau}{M_{1}+\hat{\Lambda}_{1}}, \\
R_{1}=-\sqrt{\tilde{\alpha}}, \tilde{\alpha}=\frac{\tilde{\Psi}_{33}}{\tilde{\Psi}_{11}}>0, \\
R_{2}=-\frac{\tau}{M_{1}}, \\
R_{3}=-1+\varepsilon R_{3}^{(1)}+\ldots, \\
R_{4}=-1+\varepsilon R_{4}^{(1)}+\ldots, \\
R_{5}=-\frac{\tau_{0}}{1+M_{1}}-\varepsilon \frac{\tau_{1}}{1+M_{1}}+\ldots ;
\end{gathered}
$$

b) if $M_{1}+\hat{\Lambda}_{1} \leq 0$, then the roots with the desired property are determined by expressions for $R_{1}, \ldots, R_{5}$ (see ( $(7.13)$ ). Here $R_{3,4}^{(1)}= \pm \sqrt{\tilde{\chi}_{0}}$, and

$$
\tilde{\chi}_{0}=\frac{\bar{l}^{2}\left(1-\bar{l}^{2}-\hat{\beta}^{2}\right)-\left(\bar{l}^{2}-1\right)\left(\hat{B} \hat{\lambda}^{2}-\hat{\sigma}\right)}{4 \hat{N}\left(\bar{l}^{2}-1\right)}, \bar{l}=\tau_{0}-M_{1},
$$

where the constants $M_{1}\left(0<M_{1}<1\right)$ and $\hat{N}>0$ are described above.

Taking into account the foregoing, we finally seek a particular solution of problem (7.1) (7.3) in the form

$$
\left(\begin{array}{c}
E_{1 \infty} \\
E_{3 \infty}
\end{array}\right)(t, x, z)=\exp \{n(\tau t+i z)\}\left(\begin{array}{l}
\mathcal{E}_{1 \infty} \\
\mathcal{E}_{3 \infty}
\end{array}\right) \exp \left(n r_{+} x\right), x<0
$$




$$
\begin{aligned}
& \left(\begin{array}{c}
w \\
s \\
v_{1} \\
v_{3} \\
\tilde{p} \\
E_{1} \\
E_{3} \\
\rho_{e}
\end{array}\right)(t, x, z)=\exp \{n(\tau t+i z)\}\left\{\left(\begin{array}{c}
\varepsilon \tilde{W}_{0}^{(0)} \\
S^{(0)} \\
V_{1}^{(0)} \\
V_{3}^{(0)} \\
\tilde{\mathcal{P}}^{(0)} \\
\mathcal{E}_{1}^{(0)} \\
\mathcal{E}_{3}^{(0)} \\
R_{e}^{(0)}
\end{array}\right) \exp \left(n R_{0} x\right)\right. \\
& +\left(\begin{array}{c}
0 \\
0 \\
0 \\
0 \\
0 \\
\mathcal{E}_{1}^{(1)} \\
\mathcal{E}_{3}^{(1)} \\
0
\end{array}\right) \exp \left(n R_{1} x\right)+\left(\begin{array}{c}
\varepsilon \tilde{W}_{0}^{(2)} \\
S^{(2)} \\
0 \\
0 \\
0 \\
0 \\
0 \\
0
\end{array}\right) \exp \left(n R_{2} x\right) \\
& \left.+\sum_{j=3}^{5}\left(\begin{array}{c}
\varepsilon \tilde{W}_{0}^{(j)} \\
0 \\
V_{1}^{(j)} \\
V_{3}^{(j)} \\
\tilde{\mathcal{P}}^{(j)} \\
0 \\
0 \\
0
\end{array}\right) \exp \left(n R_{j} x\right)\right\}, x>0 \\
& f(t, x, z)=F \exp \{n(\tau t+i z)\}, \\
& \omega(t, x, z)=\Omega \exp \{n(\tau t+i z)\} .
\end{aligned}
$$

In case b) the underlined item in (7.16) is absent.

For finding the constants

$$
\begin{gathered}
F, \Omega, \mathcal{E}_{1 \infty} \mathcal{E}_{3 \infty}, \tilde{W}(0)_{0}, S(0), V(0)_{1}, V(0)_{3}, \tilde{\mathcal{P}}(0), \mathcal{E}(0)_{1}, \mathcal{E}(0)_{3}, R_{e}(0), \\
\mathcal{E}(1)_{1}, \mathcal{E}(1)_{3}, \tilde{W}(2)_{0}, S(2), \tilde{W}(j)_{0}, V(j)_{1}, V(j)_{3}, \tilde{\mathcal{P}}(j), j=3,4,5
\end{gathered}
$$

we have a linear algebraic system of 27 equations, but the number of unknowns is 28 . Therefore, this system is underdetermined and, therefore, always has a nontrivial solution (see [18]).

Thus, we have constructed a nontrivial particular solution of problem (7.1)-(7.3). As is known (see [15), this means that we have proved the ill-posedness of the linear problem (7.1) - (7.3) and, therefore, the instability of shock waves. 
Now we pass to case b). For this case we obtain a linear algebraic system of 19 equations, and the number of unknowns is 19 as well. This system has the equations

$$
\begin{gathered}
i \mathcal{E}_{1 \infty}-r_{+} \mathcal{E}_{3 \infty}=0, \\
i \mathcal{E}_{1}^{(1)}-R_{1} \mathcal{E}_{3}^{(1)}=0, \\
\tilde{q}^{(2)} \tilde{W}_{0}^{(2)}+i \varepsilon^{2} \hat{\Sigma} S^{(2)}=0, \tilde{q}^{(2)}=\tilde{q}\left(R_{2}\right), \\
\hat{l}_{j} \tilde{W}_{0}^{(j)}+V_{3}^{(j)}=0, \\
\hat{l}_{j} V_{1}^{(j)}+R_{j} \tilde{\mathcal{P}}^{(j)}=0, \\
\hat{l}_{j} \tilde{\mathcal{P}}^{(j)}+R_{j} V_{1}^{(j)}+i \hat{\beta} V_{3}^{(j)}=0, \hat{l}_{j}=\tau+M_{1} R_{j}, j=3,4,5, \\
\tilde{W}_{0}^{(2)}+\sum_{j=3}^{5} \tilde{W}_{0}^{(j)}=0, \\
n \tau F=\frac{\mu}{\hat{\mu}} \sum_{j=3}^{5} \tilde{\mathcal{P}}^{(j)}, \\
\sum_{j=3}^{5}\left(V_{1}^{(j)}+d M_{1} \tilde{\mathcal{P}}^{(j)}\right)=0, \\
{\left[\left(M_{1}^{2}-\hat{\sigma}\right) \varepsilon^{2}-\hat{N}\right] \sum_{j=2}^{5} R_{j} \tilde{W}_{0}^{(j)}} \\
\tilde{E}_{3 \infty}^{5} \sum_{j=2}^{(j)}-M_{1} \Theta \varepsilon^{2} \mathcal{E}_{3}^{(1)}+M_{1} \bar{\mu} i \varepsilon F=0, \\
\mathcal{E}_{1}^{(1)}-\bar{A} \mathcal{E}_{1 \infty}=0, \\
\mathcal{E}_{j}^{(1)}+W_{E} i n F .
\end{gathered}
$$

To simplify subsequent calculations we write down system (17.17) assuming that

$$
\tilde{\Psi}_{11}=\tilde{\Psi}_{11 \infty}=\frac{1}{2} \text {. }
$$

Combining the two first and the two last equations of system (17.18) we find term $\mathcal{E}_{3}^{(1)}$ :

$$
\mathcal{E}_{3}^{(1)}=-\frac{W-\bar{A} W_{\infty}}{1-\frac{R_{1}}{r_{+}}} i n F
$$

In view of (7.19), the fourth equation of system (7.18) from the bottom becomes

$$
\left[\left(M_{1}^{2}-\hat{\sigma}\right) \varepsilon^{2}-\hat{N}\right] \sum_{j=2}^{5} R_{j} \tilde{W}_{0}^{(j)}+\hat{N} \sum_{j=2}^{5} R_{j}^{3} \tilde{W}_{0}^{(j)}+M_{1} \hat{\mu} \Theta_{1} \varepsilon^{2} i n F=0,
$$


where

$$
\Theta_{1}=1+\frac{W \frac{R_{1}}{r_{+}}-\bar{A} W_{\infty}}{\hat{\mu}\left(1-\frac{R_{1}}{r_{+}}\right)} \Theta .
$$

Taking into account (7.20), the remaining equations of system (7.18) can be reduced to the following linear algebraic system of three equations:

$$
\begin{gathered}
\sum_{j=3}^{5}\left\{\nu \hat{\beta} \hat{l}_{j}^{2} \hat{\Sigma} \varepsilon^{2}+\tilde{q}^{(2)}\left(\hat{l}_{j}^{2}-R_{j}^{2}\right)\right\} \hat{\mathcal{P}}^{(j)}=0, \hat{\mathcal{P}}^{(j)}=\frac{\tilde{\mathcal{P}}^{(j)}}{\hat{l}_{j}} \\
\sum_{j=3}^{5} \hat{l}_{j}\left(d M_{1} \hat{l}_{j}-R_{j}\right) \hat{\mathcal{P}}^{(j)}=0 \\
\sum_{j=3}^{5}\left\{\tau\left[\hat{N}\left(R_{j}^{2}+R_{2} R_{j}+R_{2}^{2}-1\right)+\varepsilon^{2}\left(M_{1}^{2}-\hat{\sigma}\right)\right]\left(\hat{l}_{j}^{2}-R_{j}^{2}\right)\right. \\
\left.-\mu \hat{\beta} \hat{l}_{j} \Theta_{1} M_{1}^{2} \varepsilon^{2}\right\} \hat{\mathcal{P}}^{(j)}=0 .
\end{gathered}
$$

Equating the determinant of system (7.21) to zero, we obtain a so-called "dispersion" relation. Expanding this relation into the series of $\varepsilon$, we write down the coefficient by the first degree of $\varepsilon$ and equate it to zero:

$$
\frac{2 \hat{N}^{2}\left(d M_{1}-1\right)}{M_{1}^{2}\left(1+M_{1}\right)^{2}} \tau_{0}^{3}\left(\tau_{0}+2 M_{1}\right)\left(\bar{l}^{2}-1\right) \sqrt{\tilde{\chi}_{0}}=0,
$$

where the term $\tilde{\chi}_{0}$ is defined by formula (7.14). Assuming that $\tilde{\chi}_{0}=0$, i.e.,

$$
\bar{l}^{4}+\left(\hat{B} \hat{\lambda}^{2}-\hat{\sigma}+\hat{\beta}^{2}-1\right) \bar{l}^{2}-\left(\hat{B} \hat{\lambda}^{2}-\hat{\sigma}\right)=0,
$$

we find the roots of this equation:

$$
\bar{l}_{2}=\frac{1+\hat{\sigma}-\hat{\beta}^{2}-\hat{B} \hat{\lambda}^{2} \pm \sqrt{\left(\hat{B} \hat{\lambda}^{2}+\hat{\beta}^{2}-\hat{\sigma}-1\right)+4\left(\hat{B} \hat{\lambda}^{2}-\hat{\sigma}\right)}}{2}=æ_{ \pm} .
$$

If $\hat{\sigma}<\hat{B} \hat{\lambda}^{2}$, then taking $\tau_{0}=M_{1}+\sqrt{\mathscr{\varkappa}_{+}}$, we thus finish the construction of a Hadamardtype ill-posedness example for case $b$ ).

8. Final remarks. The analysis in the previous section shows that shock waves in the given model of layered structures are unstable. The proof of instability of shock waves followed from the construction of Hadamard-type ill-posedness examples.

Acknowledgements. The authors are indebted to Dr. Yu. L. Trakhinin for help in the preparation of the manuscript of this paper. This work was supported by the Russian Foundation for Basic Research (the grant number 07-01-00585), the Interdisciplinary Integration Project No. 91 (2009) for Basic Research of the Siberian Branch of the Russian Academy of Sciences, and Russian Federal Agency of Education, Russian Ministry of Education and Science ("Progress of Science Potential of the University Education (2009-2090)", grant no. 2.1.1/4591. 


\section{REFERENCES}

[1] Dorovsky V.N., Dorovsky S.V. A hydrodynamic model of water-oil layered systems containing gas. Math. Comput. Mod., 2002, v. 35, pp. 751-757.

[2] Dorovsky V.N., Belonosov V.S., Belonosov A.S. Numerical investigation of parametric resonance in water-oil structures containing gas. Math. Comput. Mod., 2002, v. 36, pp. 203-209. MR1925071

[3] Gogosov V.V, Polansky V.A. Electrohydrodynamics: Problem and applications, fundamental equations, discontinuous solutions. Fluid mechanics (Science and technique resume), 1976, 10, pp. 5-85 (Russian).

[4] Dorovsky S.V., Dorovsky V.N. Possibility of electrometry at the investigation on stability of shock waves in layered structures. Geology and Geophysics, 2006, v. 47(7), pp. 892-901 (Russian).

[5] Dorovsky S.V., Dorovsky V.N., Blokhin A.M. Possibility of the methods of electrometry at the investigation on stability of shock waves in layered structures. Geology and Geophysics, v. 47, pp. 1185-1191 (Russian).

[6] Blokhin A.M., Dorovsky V.N. Problems of the mathematical simulation in theory of the multivelocity continuum. RAS, Sib. Division., United Inst. of Geology, Geophysics and Mineralogy, Inst. of Math., Novosibirsk, 1994 (Russian).

[7] Kats E.N., Lebedev V.V. Dynamics of liquid crystals. Moscow, Nauka, 1988 (Russian).

[8] Landau L.D., Lifshits E.M. Theoretical physics. v. VII. Theory of elasticity. Moscow, Nauka, 1987 (Russian). MR912888 (88m:00005)

[9] Landau L.D., Lifshits E.M. Theoretical physics. v. VI. Hydrodynamics. Moscow, Nauka, 1986 (Russian). MR850480 (87j:76001)

[10] Blokhin A.M., Dorovsky S.V. Shock waves stability in layered structures. Comput. Math Appl., 2004, v. 47, pp. 427-440. MR2048194 (2005j:35148)

[11] Blokhin A.M., Dorovsky S.V., Ovechkin E.V. Stability of shock waves in layered structures. Part 2. Comput. Math. Appl., 2004, v. 47, pp. 1379-1387. MR2070991(2005e:35236)

[12] Sedov L.N. Mechanics of continua, v.1. Moscow., Nauka, 1970 (Russian).

[13] Ovsyannikov L.V. Lectures on the foundations of the gas dynamics. Moscow, Nauka, 1981 (Russian). MR665918 (84f:76041)

[14] Blokhin A.M., Merazhov I.Z., Trakhinin Yu.L. Investigation on stability of electrohydrodynamic shock waves. Le Matematiche, vol. LII (1997)-Fasc. I, pp. 87-114. MR1624964 (99e:76125)

[15] Blokhin A.M. Energy integrals and their applications to gas dynamics problems. Nauka, Sibirsk. Otdel., Novosibirsk, 1986 (Russian). MR888816 (88m:76049)

[16] Blokhin A.M. The elements of the theory of hyperbolic systems and equations. Novosibirsk. Gos. Univ., Novosibirsk, 1995 (Russian).

[17] Godunov S.K. Equations of mathematical physics. Nauka, Moscow, 1979 (Russian). MR548574 (80i:35001)

[18] Bellman R. Introduction to matrix analysis. McGraw-Hill Book Company, Inc., New York, 1960. MR0122820 (23:A153) 